\title{
Variability of the Lagrangian turbulent diffusion in the lower stratosphere
}

\author{
B. Legras ${ }^{1}$, I. Pisso ${ }^{1}$, G. Berthet ${ }^{2}$, and F. Lefèvre ${ }^{2}$ \\ ${ }^{1}$ Laboratoire de Météorologie Dynamique, UMR8539, Paris, France \\ ${ }^{2}$ Service d'Aéronomie, UMR7620, Paris, France
}

Received: 28 October 2004 - Published in Atmos. Chem. Phys. Discuss.: 15 December 2004

Revised: 13 April 2005 - Accepted: 30 May 2005 - Published: 22 June 2005

\begin{abstract}
Ozone and nitrous oxide are measured at high spatial and temporal resolution by instruments flying on the ER2 NASA research aircraft. Comparing the airborne transects to reconstructions by ensemble of diffusive backward trajectories allows estimation of the average vertical Lagrangian turbulent diffusion experienced by the air parcels. The resulting estimates show large Lagrangian diffusion of the order of $0.1 \mathrm{~m}^{2} \mathrm{~s}^{-1}$ in the surf zone outside the polar vortex and smaller values of the order of $0.01 \mathrm{~m}^{2} \mathrm{~s}^{-1}$ inside. Locally, large variation of Lagrangian diffusion occurs over mesoscale distances. It is found that high temporal resolution ( $3 \mathrm{~h}$ or less) is required for off-line transport calculations and that the reconstructions are sensitive to spurious motion in standard analysed winds.
\end{abstract}

\section{Introduction}

The distribution of chemical compounds in the atmosphere exhibits a large range of variability that is partly due to transport. This is particularly true in the lower stratosphere for species, like nitrous oxide $\mathrm{N}_{2} \mathrm{O}$ which have no sources or sinks in this region. Ozone is chemically reactive in the lower stratosphere but its lifetime exceeds several weeks except in the regions where chlorine is activated within the winter polar vortex; that is long enough for transport to be effective.

In the lower stratosphere, vertical motion is limited by stratification to be of the order of $1 \mathrm{~K} /$ day in potential temperature. Hence it takes about 3 weeks to travel over a vertical distance of $1 \mathrm{~km}$ and transport is mostly dominated by horizontal motion. With a vertical shear $\Lambda \approx 210^{-3} \mathrm{~s}^{-1}$ and a horizontal strain $\gamma \approx 10^{-5} \mathrm{~s}^{-1}$, a compact cloud of particles is first dispersed by vertical shear during a few hours until it reaches an equilibrium slope of $\Lambda / \gamma \approx 200$ within about

Correspondence to: B. Legras

(legras@1md.ens.fr) one day after which dispersion is mainly due to the horizontal strain (Haynes and Anglade, 1997). Under the repeated action of strain and foldings due to the nonlinearity of the flow, tracers are stirred and form a number of sloping sheets which are observed as laminae in vertical soundings and aircraft transects. The core of intense jets, such as in the stratospheric polar vortex, acts as a transport barrier and is often associated with strong tracer gradient.

The proof of this concept is provided by the ability to reconstruct the small-scale distribution of tracers by advection methods using analysed winds (Waugh et al., 1994; Sutton et al., 1994; Mariotti et al., 1997). The basis of these methods is that layer-wise tracer advection in the stratosphere is dominated by the large scales of motion which are sufficiently well resolved by operational analysed winds provided by the major weather centers. This flow regime is known as the Batchelor regime in fluid dynamics (Haynes and Vanneste, 2004; Falkovich et al., 2001). Hence, the tracer structures at scales smaller than the analysed winds are to some extent predictable by integrating the advection equation backward in time (Methven and Hoskins, 1999). There is no contradiction here: the information about unresolved tracer scales is fully contained in the time series of wind fields.

Reconstruction by pure advection generates, however, an endless growing number of structures in the tracer field as the reconstructed time increases. In fact, mixing performed by small-scale turbulence, not represented in the analysed winds, limits the smallest scale that tracer sheets may sustain. Assuming that the small-scale turbulence is roughly isotropic, vertical mixing is the most effective process in providing a cut-off scale for the tracer sheets, the vertical cut-off being converted into an horizontal cut-off by the slope factor.

Small-scale turbulence is due to a number of instabilities, either local instabilities of the wind profile or breaking of vertically propagating gravity waves. It is believed to be patchy in space and time. Although some elaborate representations are available (e.g., Vanneste and Haynes, 2000;

(C) 2005 Author(s). This work is licensed under a Creative Commons License. 
Konopka et al., 2004), our goal here is not to test a particular parametrization but to provide an independent estimate of unresolved turbulent motion as an ordinary vertical diffusion $D$.

The literature exhibits a variety of estimates of $D$ ranging from $5 \mathrm{~m}^{2} \mathrm{~s}^{-1}$ to $0.001 \mathrm{~m}^{2} \mathrm{~s}^{-1}$ The largest values are obtained from radar measurements assuming homogeneous turbulence and near critical Richardson number (Woodman and Rastogi, 1984; Fukao et al., 1994; Nastrom and Eaton, 1997). These values are contradicted by recent estimates from high resolution balloon data (Alisse et al., 2000) and by studies of large-scale advective stirring (Waugh et al., 1997; Balluch and Haynes, 1997) that provide values in the lower part of the range, of the order of $0.01 \mathrm{~m}^{2} \mathrm{~s}^{-1}$ or less.

Both estimates of Waugh et al. (1997) and Balluch and Haynes (1997) were based, like the present study, on the dominating layer-wise motion in the stratosphere to generate tracer sheets. From the assumption that tracer structures are sloping sheets, Balluch and Haynes reduced locally the advection and the diffusion of a tracer to a one dimensional equation projected on an evolving gradient direction. They estimated an upper limit on vertical diffusivity by reconstructing several laminae selected from $\mathrm{N}_{2} \mathrm{O}$ airborne measurements, varying the diffusivity until the reconstruction best agrees with the observations. In this study, we go one step further by removing any assumption about tracer distribution and using a powerful method to solve locally the advection-diffusion problem.

This new approach has been introduced in Legras et al. (2003) to study vertical diffusivity from the reconstruction of vertical ozone profiles. The conclusion of this study was to put an upper limit of $0.1 \mathrm{~m}^{2} \mathrm{~s}^{-1}$ for the vertical diffusivity in the lower stratosphere mid-latitude surf zone during winter. The possibility to test smaller values of the diffusivity was, however, impaired by the limited vertical resolution of ozone soundings with standard chemical sondes that is of the order of $100 \mathrm{~m}$ if we stay on the optimistic side. Using the slope factor 200, the equivalent horizontal resolution of ozone soundings does not exceed $20 \mathrm{~km}$, while airborne tracer measurements are currently performed with resolution under $1 \mathrm{~km}$ for species like $\mathrm{O}_{3}, \mathrm{CH}_{4}$ or $\mathrm{N}_{2} \mathrm{O}$ that can be measured at high frequency of one to a few Hertz. Hence, airborne measurements resolve at least 20 times better the small-scale sloping structure than standard ozone soundings. The above motivates the present study which extends Legras et al. (2003) by analysing airborne transects collected by the instruments on board the NASA ER-2 during the SOLVE campaign in the Arctic in January-March 2000.

Section 2 presents the method used for the Lagrangian reconstructions based on the advective-diffusive equation. Section 3 describes the data and trajectory calculations used in this study. Section 4 demonstrates that diffusive reconstructions of a tracer are strikingly stable over a large range of reconstruction times. Section 5 defines the roughness criterion used to fit the vertical diffusion in this study. Section 6 discusses the reconstructions of the most significant SOLVE flights and the best fitting diffusivities. Section 7 discusses local structures. Section 8 discusses the relation between diffusivity and dispersion. Section 9 shows the spurious effects of under-resolving the temporal variations of the wind. Finally, Sect. 10 offers further discussion, including a comparison with previous results of Balluch and Haynes (1997), and conclusions.

\section{Diffusive reconstruction}

The standard reconstruction method for the mixing ratio of a tracer at time $t_{0}$ within a given domain $\mathcal{D}$ consists in finding the location, at an earlier time $t_{0}-\tau$, of the parcels filling $\mathcal{D}$ at time $t_{0}$, and to attribute a mixing ratio to each parcel according to the tracer mixing ratio at its initial location at time $t_{0}-\tau$. The initial location is found by backward integration of the particle advection equation $d \boldsymbol{x} / d t=\boldsymbol{u}(\boldsymbol{x}, t)$ where the wind $\boldsymbol{u}(\boldsymbol{x}, t)$ is interpolated in time and space from the analysed winds provided by operational weather centers. The main interest of this calculation is that the reconstructed field at time $t_{0}$ gives access to much smaller scales than the initialisation field used at time $t_{0}-\tau$. In many previous studies, the tracer was potential vorticity (PV) and it was initialised according to the analysis from weather centers. The value of this approach has been demonstrated by comparing the reconstructed PV with observations of tracers, either in the stratosphere with aerosols and ozone (Waugh et al., 1994; Sutton et al., 1994; Mariotti et al., 1997; Orsolini et al., 2001) or in the troposphere with water vapour (Appenzeller et al., 1996). However, PV can hardly be measured by in situ instruments and cannot be assumed to correlate perfectly with any measurable tracer. Hence, recent efforts have been devoted to the direct reconstruction of observable chemical fields. The tracer distribution at time $t_{0}-\tau$ is then provided, with a crude resolution, either by a chemical transport model (Legras et al., 2003) or by satellite observations (Orsolini et al., 2001).

The standard reconstruction method is purely advective and fully deterministic. It fails to take into account any diffusive process and generates a number of small-scale structures that increases exponentially with the reconstruction time $\tau$. It is usually considered that, according to the resolution of initial fields, reconstructions should be performed over durations of 10 to 20 days in the lower stratosphere (Methven and Hoskins, 1999; Waugh and Plumb, 1994) beyond which the number of spurious structures pervades the results.

In our approach, as sketched in Fig. 1, diffusion is taken into account by splitting the parcel at time $t_{0}$ into $N$ particles which are all advected backward in time by the equation

$$
\delta \boldsymbol{x}=\boldsymbol{u}(\boldsymbol{x}, t) \delta t+\boldsymbol{k} \delta \eta(t),
$$

where $\boldsymbol{k}$ is the vertical unit vector and $\delta \eta(t) \equiv w(t) \delta t$ is the product of a white noise process $w(t)$ by the time step $\delta t$. The 


\section{M}

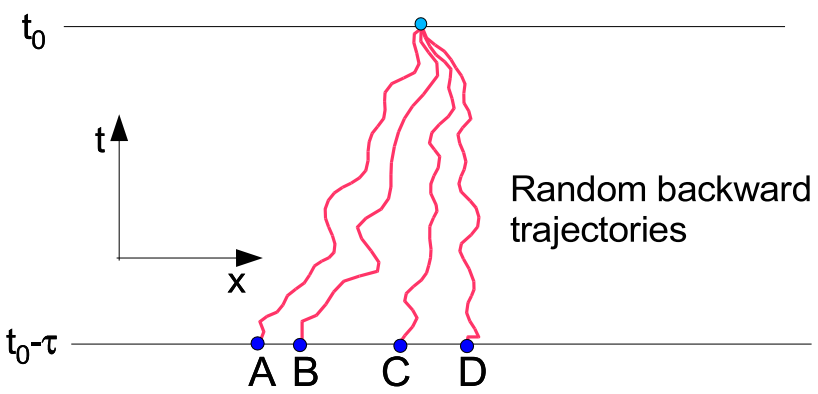

Fig. 1. Sketch of the trajectories of the transported and diffused particles meeting in point $M$ at time $t_{0}$ after travelling from their initial locations $(A, B, C, D)$ at time $t_{0}-\tau$. The mixing ratio in $M$ and $t_{0}$ is reconstructed from averaging the mixing ratios in $(A, B, C, D)$ and $t_{0}-\tau$.

white noise process is without memory (i.e. it is $\delta$-correlated in time), and with a zero mean. In the limit $\delta t \rightarrow 0$ and after statistical average over a large number of particles, this is equivalent to adding a diffusion $D$ to transport with

$$
D=\frac{1}{2}<w^{2}>\delta t
$$

In order to ensure that vertical velocities are bounded, we use a white noise based on a random variable $r$ that is uniformly distributed over the interval $[-\sqrt{3}, \sqrt{3}]$ with zero mean and unit variance. Applying Eq. (2), the random process is then $\delta \eta=r \sqrt{2 D \delta t}$ with a new drawing of $r$ at each time step and for each particle. Actually, we use a timestep of $18 \mathrm{~s}$ for the random term which is fifty times smaller than the time-step for advection in order to enhance statistical convergence.

The reconstructed mixing ratio of the parcel is the average of the mixing ratios of the $N$ particles initialised at time $t_{0}-\tau$.

The method just described is directly related to the solution of the advective-diffusive equation for the mixing ratio $\chi$ of a passive tracer

$$
\chi(\boldsymbol{x}, t)=\int \rho(\boldsymbol{y}, s) G(\boldsymbol{x}, t ; \boldsymbol{y}, s) \chi(\boldsymbol{y}, s) d^{3} \boldsymbol{y},
$$

where $G(\boldsymbol{x}, t ; \boldsymbol{y}, s)$ is the Green function describing the probability of a particle that was in $\boldsymbol{y}$ at time $s$ to be in $\boldsymbol{x}$ at time $t$, and $\rho$ is air density. The Green functions satisfies the two equations (Morse and Feschbach, 1953)

$$
\begin{aligned}
\frac{\partial G}{\partial t}+\boldsymbol{u}(\boldsymbol{x}, t) \cdot \nabla_{\boldsymbol{x}} G & -\frac{D}{\rho(\boldsymbol{x}, t)} \nabla_{\boldsymbol{x}} \rho(\boldsymbol{x}, t) \nabla_{\boldsymbol{x}} G \\
& =\frac{1}{\rho(\boldsymbol{y}, s)} \delta(t-s) \delta(\boldsymbol{y}-\boldsymbol{x}), \\
\frac{\partial G}{\partial s}+\boldsymbol{u}(\boldsymbol{y}, s) \cdot \nabla_{\boldsymbol{y}} G & +\frac{D}{\rho(\boldsymbol{y}, t)} \nabla_{\boldsymbol{y}} \rho(\boldsymbol{y}, t) \nabla_{\boldsymbol{y}} G \\
& =\frac{1}{\rho(\boldsymbol{x}, t)} \delta(t-s) \delta(\boldsymbol{y}-\boldsymbol{x}) .
\end{aligned}
$$

Here, the derivatives are taken with respect to the final coordinates $\boldsymbol{x}$ and $t$ for the first equation and with respect to the initial coordinates $\boldsymbol{y}$ and $s$ for the second one. The statistical average of mixing ratio over random backward trajectories is equivalent to solving Eq. (5) up to neglecting the variations of $\rho$ over turbulent diffusive scales. The negative sign in front of the diffusive term on the r.h.s. of Eq. (5) means that this equation is well-posed for backward integration in time. For a more detailed discussion, see Holzer and Hall (2000) and Issartel and Baverel (2003).

Notice that the random process entering the advection equation has been described here assuming that timestepping is performed using a simple Euler scheme. More sophisticated numerical schemes require adequate transformations.

\section{Data and trajectory processing}

Data have been collected by NASA ER-2 aircraft during SOLVE campaign from January to March 2000. The ER2 is equipped with a number of instruments performing in situ measurements of chemical tracers. We have used the unified nitrous oxide data combining the measurements of three instruments (ACATS, ARGUS, ALIAS) at $0.33 \mathrm{~Hz}$ with a resulting relative precision of 3 ppbv $(1.5 \%)$ at flight level (Hurst et al., 2002) and the NOAA ozone photometer with $1 \mathrm{~Hz}$ frequency and a relative precision of $10 \mathrm{ppbv}$ (0.5\%) (Proffitt et al., 1989). During the SOLVE campaign, eleven flights were performed from Kiruna (67.83 N, $20.42 \mathrm{E}$ ) which was then located inside the polar vortex. We have processed ten of the flights during which all the relevant instruments were operating. Except for two dates on 27 January 2000 and 11 March 2000, the ER-2 flights were performed entirely inside the polar vortex and measured fairly homogeneous mixing ratios of $\mathrm{N}_{2} \mathrm{O}$ and $\mathrm{O}_{3}$ over constant pressure level legs, indicating that the vortex was well mixed and homogenized during this period. Indeed, the Arctic winter 2000 has been remarkably cold with only a minor warming in early February and no major warming until mid-March (Rex et al., 2002). We have also used the transit flights from Kiruna to California on 16 March 2000 and 18 March 2000 during which the instruments were operating in order to compare turbulent diffusivity in the vortex and in the mid-latitude surf zone. 


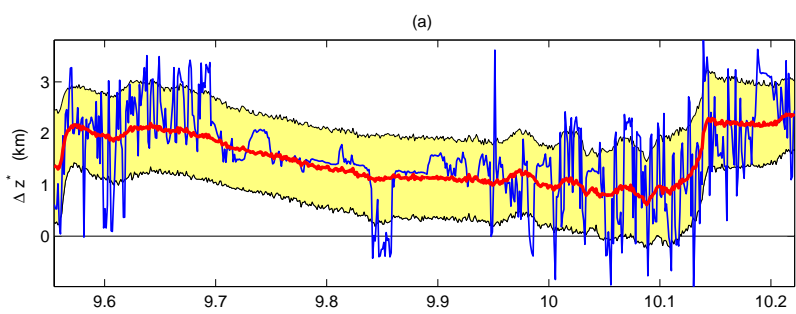

(b)

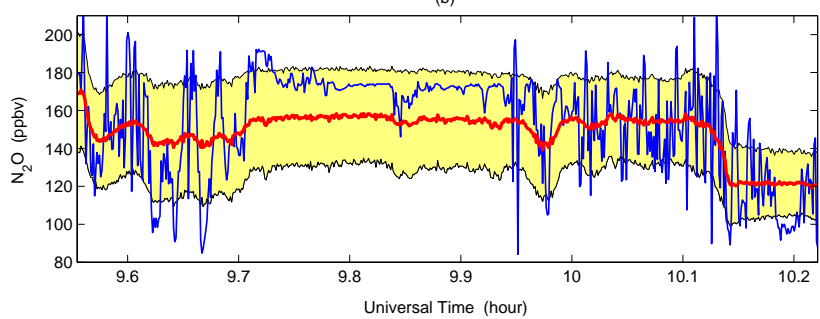

Fig. 2. (a): Vertical displacement $\Delta z^{*}$ of the particles with respect to their launch on the flight track, where $z^{*}=H_{0} \ln \left(p_{0} / p\right)$ $\left(p_{0}=1000 \mathrm{hPa}, H_{0}=5850 \mathrm{~m}\right)$. In red: average displacement for the diffusive motion with $D=0.01 \mathrm{~m}^{2} \mathrm{~s}^{-1}$. The yellow region spans one standard deviation from the average. In blue: displacement for a single deterministic particle with no diffusion. Results are shown for backward integration over $\tau=38$ days and one portion of the 27 January 2000 flight. (b): Same as (a) but for the reconstructed $\mathrm{N}_{2} \mathrm{O}$ mixing ratio.

Reverse integrations of particles trajectories initialised along each transect have been performed with TRACZILLA, a modified version of the trajectory code FLEXPART (Stohl et al., 2002) which uses ECMWF (European Center for Medium range Weather Forecast) winds at $1^{\circ}$ horizontal resolution and on 60 hybrid levels with 3 -h resolution obtained by combining analysis available every $6 \mathrm{~h}$ with first guesses at intermediate times. The modifications from FLEXPART advection scheme consists mainly in discarding the intermediate terrain following coordinate system and in performing a direct vertical interpolation of winds, linear in log-pressure, from hybrid levels. The vertical velocities used in this study are computed by the FLEXPART preprocessor using a mass conserving scheme in the hybrid ECMWF coordinates. A small correction due to a missing term in FLEXPART has been introduced but has virtually no impact. The model uses a fixed time step of $\delta t=900 \mathrm{~s}$. Halving it has no impact on our results. Unless stated differently, the reconstruction are performed by releasing $N=1000$ particles every $4 \mathrm{~s}$ along the flight track that is about with the same frequency than the $\mathrm{N}_{2} \mathrm{O}$ measurements. We use the GPS ER-2 data and the onboard pressure sensor to locate the launching point, respectively horizontally and vertically. By launching the particles exactly on the flight track and also by performing a partial first time step to the nearest discretized time of the model, we take into account the short-time fluctuations in flight altitude with which tracer fluctuations are correlated (Sparling and Bacmeister, 2001).
Assignment of $\mathrm{N}_{2} \mathrm{O}$ and $\mathrm{O}_{3}$ was performed at $t_{0}-\tau$ location from three-dimensional fields produced by REPROBUS (REactive Processes Ruling the Ozone BUdget in the Stratosphere). REPROBUS is a three-dimensional chemicaltransport model (CTM) with a comprehensive treatment of gas-phase and heterogeneous chemical processes in the stratosphere (Lefèvre et al., 1994, 1998). Long-lived species, including ozone, are transported by a semi-Lagrangian scheme (Williamson, 1989) forced by the 6-hourly ECMWF wind analysis. The model is integrated on 42 hybrid pressure levels that extend from the ground up to $0.1 \mathrm{hPa}$, with a horizontal resolution of $2^{\circ}$. For the experiments presented here REPROBUS was initialised on 15 October 1999. Chemical species (including $\mathrm{N}_{2} \mathrm{O}$ ) were initialised from October zonal means obtained after a 5-year simulation driven by GCM winds. The ozone field was reinitialised on 1 December 1999 from the three-dimensional $\mathrm{O}_{3}$ analysis computed at ECMWF.

\section{Tracer reconstruction from random trajectories}

Figure 2 shows how the diffusive reconstruction differs from the standard single particle deterministic reconstruction. The particles emitted from a single point are diffused backward in time and spread spatially as seen in the upper panel. After reaching their initial locations they sample a range of $\mathrm{N}_{2} \mathrm{O}$ values. This sampling varies much less from one point to the next than any individual trajectory, thus providing a much smoother reconstruction, as seen in the lower panel. The diffusive reconstruction is not, however, just a smoothed version of the single particle reconstruction. This latter should be seen as one possible realisation among many that contributes to the statistical average of the diffusive reconstruction. The small wiggles on the diffusive reconstruction are fluctuations due to the finite sampling of $N$ particles per point. Their amplitude is proportional to $\sqrt{D / N}$.

The first panel of Fig. 3 shows $\mathrm{N}_{2} \mathrm{O}$ observed by the ER2 and as predicted by REPROBUS for the track of the 11 March 2000 flight along Norwegian coast that crossed the polar vortex edge at about 11:00 UT and crossed it back at about 13:00 UT. A sheet of polar air, marked on the figure, was crossed on both ways just outside the vortex edge. REPROBUS over predicts $\mathrm{N}_{2} \mathrm{O}$ by about $50 \%$ within the vortex but provides a good fit to the observations within the surf zone. Owing to its low spatial resolution, it fails, however, to reproduce the large gradients at the vortex edge and the sheet of polar air. The following panels of Fig. 3 show the diffusive reconstructions of $\mathrm{N}_{2} \mathrm{O}$ with $D=0.01 \mathrm{~m}^{2} \mathrm{~s}^{-1}$ and increasing values of $\tau$. For small $\tau=2$ days, the reconstructed curve does not differ strongly from REPROBUS prediction but already exhibits lower values within the vortex. At $\tau=7$ days, the vortex edge is well defined and the polar air sheet starts to emerge. The sheet is well formed at $\tau=11$ days and a number of other details emerge. By $\tau=24$ days the reconstructed 

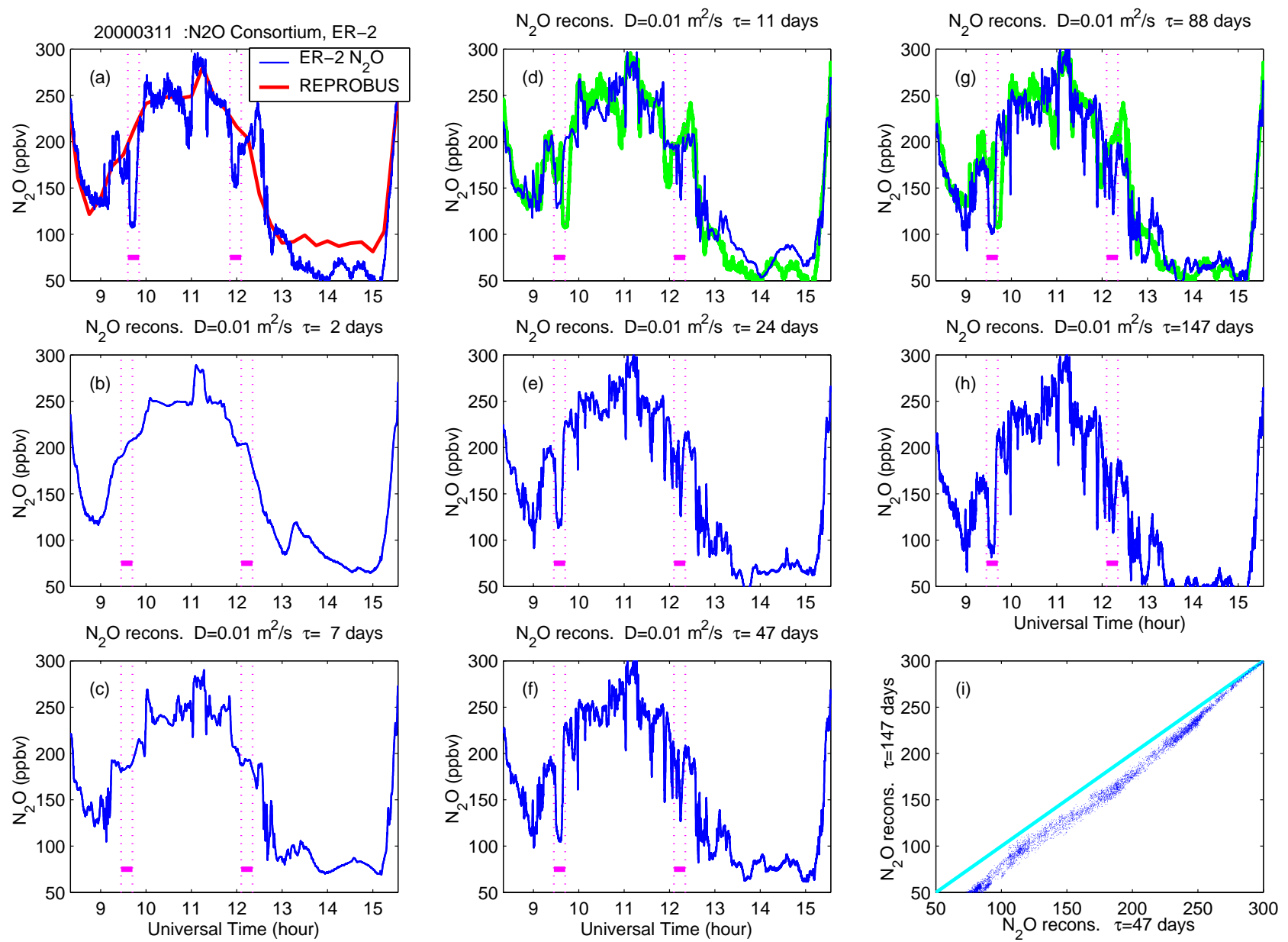

Fig. 3. (a): Observed $\mathrm{N}_{2} \mathrm{O}$ values and REPROBUS simulation for the 11 March 2000 flight. (b-h): Sequence of reconstructed transects with $D=0.01 \mathrm{~m}^{2} \mathrm{~s}^{-1}$ and increasing value of $\tau(2,7,11,24,47,88$ and 147 days). Magenta marks and dotted lines indicate the crossing of the sheet of polar vortex air. The observed values are also plotted as a thick green curve in panels (d) and (g). (i): Reconstructed $\mathrm{N}_{2} \mathrm{O}$ at $\tau=147$ days against reconstructed $\mathrm{N}_{2} \mathrm{O}$ at $\tau=47$ days.
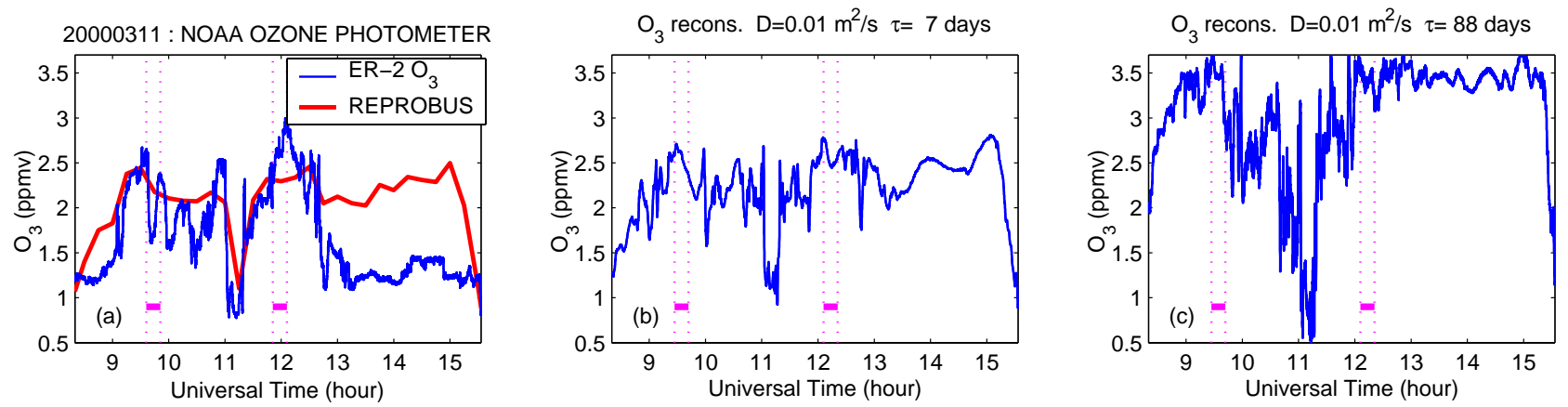

Fig. 4. Left panel: observed $\mathrm{O}_{3}$ values and REPROBUS simulation for the 11 March 2000 flight. Central and right panels: reconstructed transects with $D=0.01 \mathrm{~m}^{2} \mathrm{~s}^{-1}$ and $\tau=7$ and 147 days.

curve has reached a stable shape for all details that changes only weakly and slowly as $\tau$ increases further. For comparison, the observed values of $\mathrm{N}_{2} \mathrm{O}$ are also shown in two of the panels. The calculation comes up to $\tau=147$ days, that is more than 4 months backward, using an initialisation date before the onset of the polar vortex. Figure $3 i$ confirms the visual impression that the reconstruction evolves only weakly beyond $\tau=47$ days, the main effect being a slow decrease of $\mathrm{N}_{2} \mathrm{O}$, mostly within the polar vortex. 

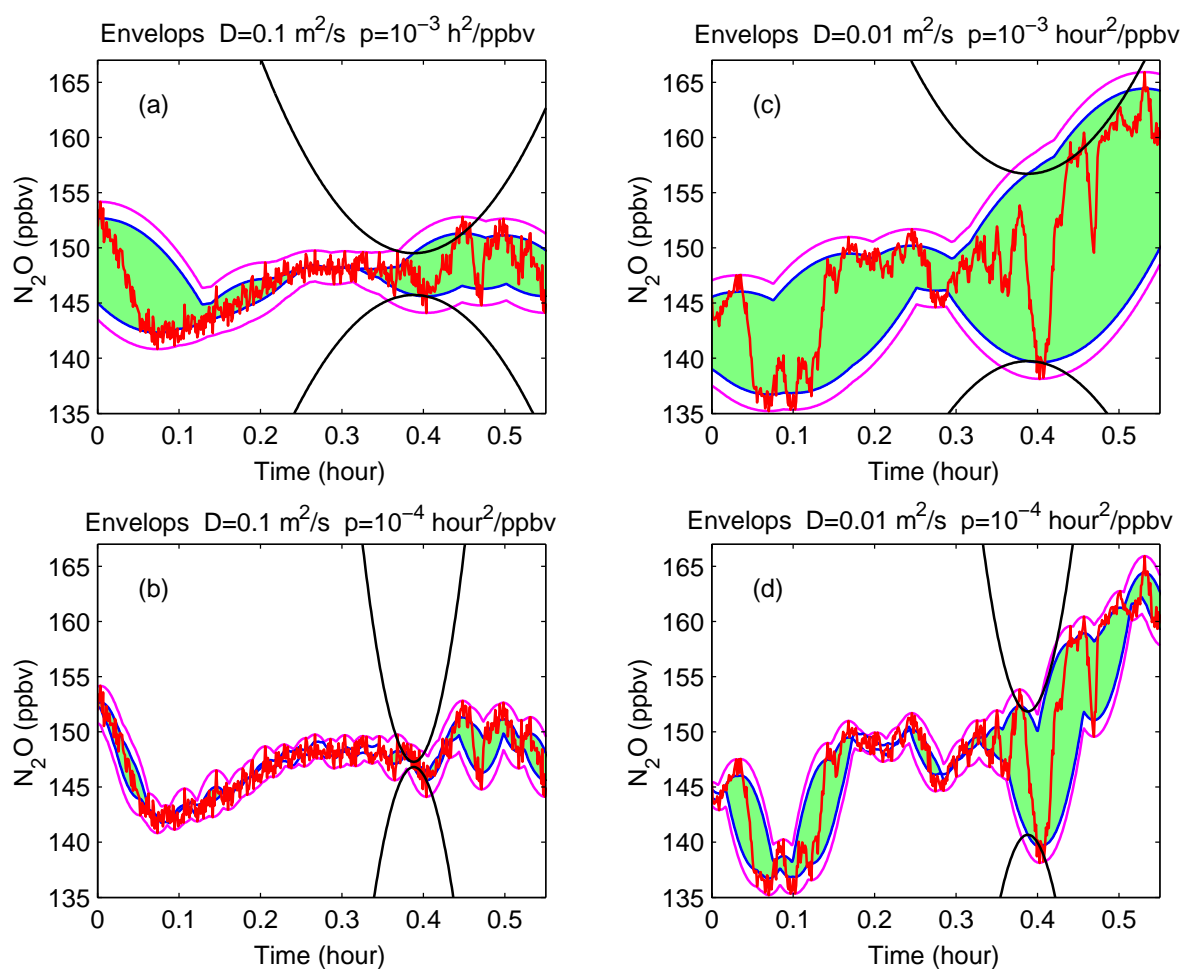

Fig. 5. The four panels show the calculation of the roughness function on $\mathrm{N}_{2} \mathrm{O}$ reconstruction for the same segment of flight using two values of diffusion $D$ and two values of $p$. (a): $D=0.1 \mathrm{~m}^{2} \mathrm{~s}^{-1}$ and $p=10^{-3} \mathrm{~h}^{2} \mathrm{ppbv}^{-1}$. (b): $D=0.1 \mathrm{~m}^{2} \mathrm{~s}^{-1}$ and $p=10^{-4} \mathrm{~h}^{2} \mathrm{ppbv}^{-1}$. (c): $D=0.01 \mathrm{~m}^{2} \mathrm{~s}^{-1}$ and $p=10^{-3} \mathrm{~h}^{2} \mathrm{ppbv}^{-1}$. (d): $D=0.01 \mathrm{~m}^{2} \mathrm{~s}^{-1}$ and $p=10^{-4} \mathrm{~h}^{2} \mathrm{ppbv}^{-1}$. The analysed curve (red) is bounded above and below by two envelope curves (magenta) tracing the tips of upper and lower osculating parabolas with parameter $p$. The positive area (green) between the two shifted envelope curves (blue) measures the roughness $\phi(p)$.

Hence, unlike single trajectory reconstruction, diffusive reconstruction is to a large extent insensitive to the reconstruction time $\tau$. This latter must be larger than an offset time required to generate large amplitude gradient from the smooth tracer field used at the initial time. The insensitivity to $\tau$ arises from the fact that Eq. (3) is valid for any time $s$ and that $\mathrm{N}_{2} \mathrm{O}$ is basically transported by REPROBUS too, albeit with a very different numerical method.

In the absence of diffusion, tracer gradients are expected to grow exponentially in time at a rate given by the average isentropic strain (Haynes and Anglade, 1997). With diffusion, the size of tracer jumps is bounded by $\sqrt{\gamma_{\max } / D}$ where $\gamma_{\max }$ is the maximum strain. Hence, the offset time is expected to depend weakly on $D$, as $\ln D$. It is also clear that the Green function dependence on $\boldsymbol{y}$ in Eq. (3) gets smoother as $\tau=t-s$ increases, and consequently that the reconstruction is only sensitive to the largest scales of the initial tracer distribution when $\tau$ is large. The intensity of gradients is mainly a property of the advection, not of the initial distribution of the tracer (Falkovich et al., 2001). The detailed discussion of the predictability of tracer gradients is deferred to another work.

Reconstructions of $\mathrm{O}_{3}$ are shown for comparison in Fig. 4. Unlike $\mathrm{N}_{2} \mathrm{O}, \mathrm{O}_{3}$ is not a conserved tracer within the po- lar vortex where it is depleted by chlorine catalyzed chemistry. During March 2000, REPROBUS has not been able to destroy enough ozone inside the polar vortex, hence the large deviation observed on the left panel of Fig. 4. This effect is accented in our calculation which does not take into account any chemistry, so that reconstructed $\mathrm{O}_{3}$ exhibits a sustained backward growth as $\tau$ increases. The fluctuations are, however, preserved like for $\mathrm{N}_{2} \mathrm{O}$. We can also argue that the slow decrease of reconstructed $\mathrm{N}_{2} \mathrm{O}$ within the polar vortex seen in Fig. 3 is due to the combined effect of sources and BrewerDobson circulation. This indicates that $\mathrm{N}_{2} \mathrm{O}$ reconstructions are basically limited by the overturning time of the BrewerDobson circulation, a fairly slight constrain in practice.

\section{Roughness}

As the goal of this study is to estimate turbulent diffusivity, we need to compare fluctuations in the observed and reconstructed tracer transects. A first way, already followed by Waugh et al. (1997) and Balluch and Haynes (1997), is to identify some structures, like the sheet of polar air already mentioned, and to adjust the diffusion to provide the best fit of reconstruction to observations. This will be used below 

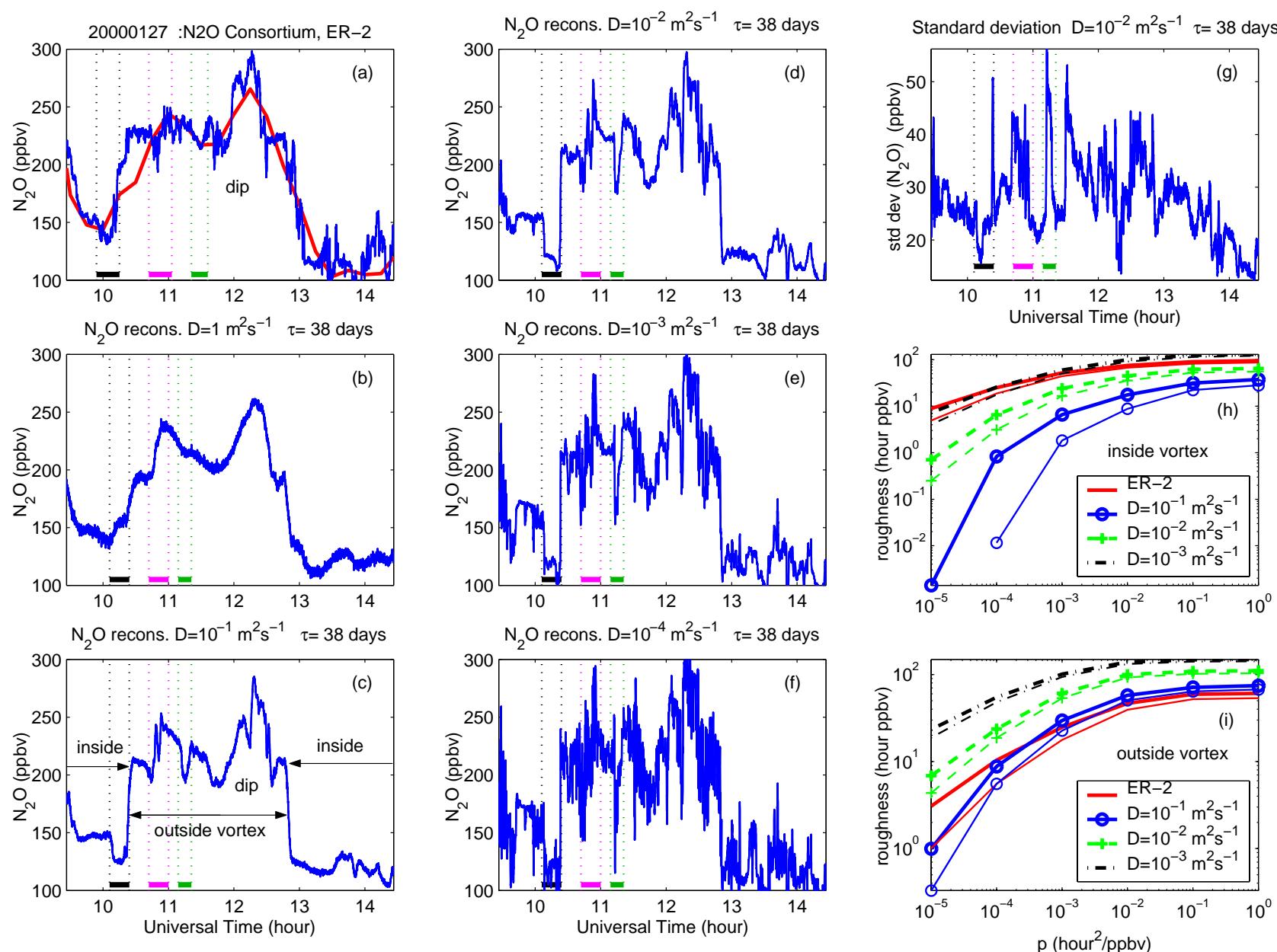

Fig. 6. Reconstructions and roughness for the 27 January 2000 flight. (a): Observed (blue) and REPROBUS (red) $\mathrm{N}_{2} \mathrm{O}$. (b-f): $\mathrm{N}_{2} \mathrm{O}$ reconstructions for $D=1,0.1,0.01,0.001$ and $0.0001 \mathrm{~m}^{2} \mathrm{~s}^{-1}$ at $\tau=38$ days. (g): Standard deviation of $\mathrm{N}_{2} \mathrm{O}$ within the $N$ particles released from each location. (h): Roughness for observed and reconstructed transects as indicated in the legend, and for the flight segment located inside the polar vortex. Thick lines: shift is $\sigma=3$ ppbv; thin lines: shift is $2 \sigma$. (i): Same as (h) but for the flight segment outside the polar vortex. Three structures are identified in the figure. The peaks in $\mathrm{N}_{2} \mathrm{O}$ and $\mathrm{O}_{3}$ near $12 \mathrm{~h}$ are due to a dip of the ER-2 and are removed from roughness analysis.

but it is not always possible to identify such structures, especially when stirring is strong. If we give up this idea, a second way consists in using a statistical measure of the fluctuations as a basis for the comparison. In Legras et al. (2003), comparisons based on spectra and increment variance have been discussed but these measures are sensitive to the small-scale noise and need to be applied to a pre-filtered signal. Legras et al. also introduced a new measure called the roughness function. We refine here this notion to take into account the small-scale instrumental noise without need to pre-filter the signal.

Our definition of roughness is provided by the following algorithm for a discrete curve described by a list of $K$ points $\left\{x_{i}, y_{i}\right\}$ with an assumed uncertainty $\pm \sigma$ :

1. For each value of $p>0$ and for each value $x_{c}=x_{i}, y_{p}^{+}\left(x_{i}\right)$ is defined as the smallest $y_{c}$ such that the parabola
$2 p\left(y-y_{c}\right)=\left(x-x_{c}\right)^{2}$ lies entirely above the curve joining the points $\left\{x_{i}, y_{i}-\sigma\right\}$.

2. Similarly, $y_{p}^{-}\left(x_{i}\right)$ is defined as the largest $y_{c}$ such that the inverted parabola, defined by turning $p$ into $-p$, lies entirely below the curve joining the points $\left\{x_{i}, y_{i}+\sigma\right\}$.

3. The two envelope curves $y_{p}^{+}\left(x_{i}\right)$ and $y_{p}^{-}\left(x_{i}\right)$ are then used to define the roughness function $\phi(p)=\frac{1}{K} \sum_{i=1}^{K} \max \left(0, y_{p}^{+}\left(x_{i}\right)-y_{p}^{-}\left(x_{i}\right)\right)$.

The reason for using a parabolic smoothing function is that arbitrary rescaling of the units of $x_{i}$ and $y_{i}$ preserves the parabolic shape, and is equivalent to multiply $p$ by a constant. For each value of $p$, the procedure produces a pair of envelope curves generated by moving the smoothing parabola in $x$ while keeping it in contact with the curve on 

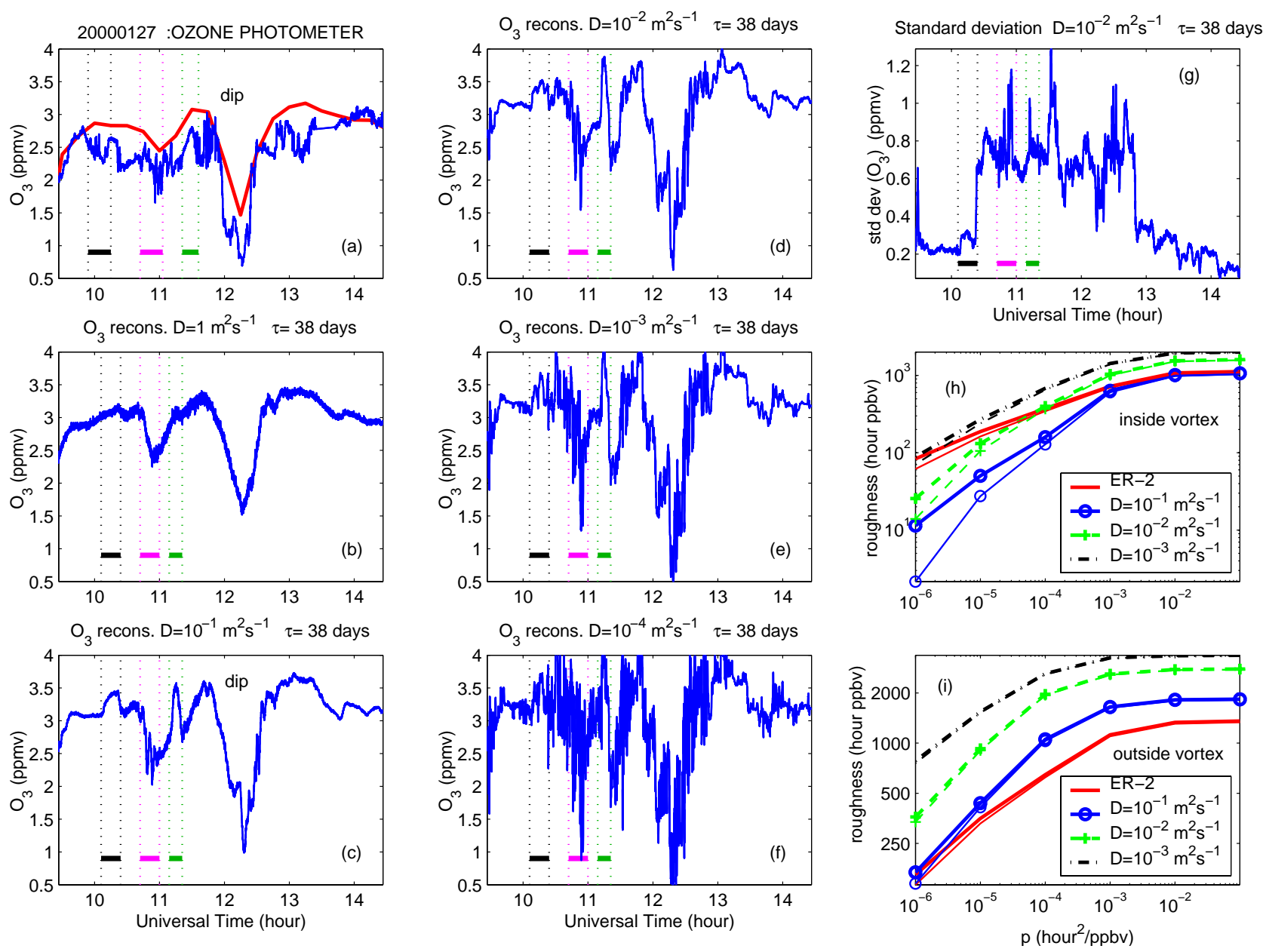

Fig. 7. Same as Fig. 6 but for $\mathrm{O}_{3}$ and $\sigma=10$ ppbv.

both side. The distance between the two envelope curves is then a measure of roughness. We do not want, however, this measure to be dominated by noise at small scale. This is accounted in steps 1 and 2 above by shifting the upper and lower envelope curves, respectively down and up, by the measurement precision $\sigma$, and by calculating the roughness function as a discretized version of the positive area between the two shifted envelope curves. Figure 5 illustrates the algorithm for two curves obtained with two values of $D$. The dependence of roughness upon scale is described by varying $p$ with multiplicative steps. It can be shown that calculating the envelope curves reduces to a Legendre transform which is performed using a fast algorithm (Lucet, 1997).

\section{Analysis of SOLVE flights}

We first present a detailed analysis of the 27 January 2000 flight which spans both the inside and the outside of the vortex and displays a number of interesting structures. Figures 6 and 7 shows the measured and reconstructed transects on 27
January 2000 for both $\mathrm{N}_{2} \mathrm{O}$ and $\mathrm{O}_{3}$ and $\tau=38$ days, with five values of $D$ ranging from a large value $D=1 \mathrm{~m}^{2} \mathrm{~s}^{-1}$, akin to radar estimates, to the molecular value $D=10^{-4} \mathrm{~m}^{2} \mathrm{~s}^{-1}$.

The large-scale variations of the observed $\mathrm{N}_{2} \mathrm{O}$ are fairly well reproduced by REPROBUS; predicted ozone is slightly larger than observed inside and outside the vortex. It appears immediately that reconstructed transects with the molecular diffusion contain much larger fluctuations than observed. On the opposite side, $D=1 \mathrm{~m}^{2} \mathrm{~s}^{-1}$ produces a much too smooth reconstruction. The edge of the polar vortex is well identified on the measured and reconstructed $\mathrm{N}_{2} \mathrm{O}$ transects but is hardly seen on the $\mathrm{O}_{3}$ transects. It shows up very well, however, on the standard deviation for reconstructed $\mathrm{O}_{3}$ which undergoes a sudden jump on the edge. The $\mathrm{N}_{2} \mathrm{O}$ standard deviation has also much larger fluctuations outside the vortex than inside and peaks on the edge, but does not exhibit a jump profile. Several structures are outlined in the measured and reconstructed transects. The first one (black) is a local $\mathrm{N}_{2} \mathrm{O}$ decrease and $\mathrm{O}_{3}$ increase along the edge, probably due to enhanced descent (Mariotti et al., 2000). The second one (magenta) is a spell of fluctuations outside the vortex and 
the third one (green) is presumably the trace of a filament expelled from the polar vortex. For all these structures, the three smallest values of $D$ reconstruct excessive amplitude with respect to the observations and $D \approx 0.1 \mathrm{~m}^{2} \mathrm{~s}^{-1}$ provides a better fit. It is also clear that the edge of the polar vortex is too steep for the three smallest values of $D$. Hence, on the edge and outside the polar vortex, the turbulent diffusion must be fairly large to account for the observed structures.

It is more difficult to associate observed and reconstructed structures inside the vortex for time $t>13: 00$ UT but the visual analysis now reveals that $D=0.1 \mathrm{~m}^{2} \mathrm{~s}^{-1}$ reconstructs a too smooth transect compared to the observation, suggesting that turbulent diffusion is smaller inside the vortex. Taking this into account, roughness has been calculated separately inside and outside the vortex (removing also the dip section) and also for two different values of the offset, one times and two times the relative precision of measurement. The two families of roughness curves displayed in Figs. 6 and 7 show that the small-scale fluctuations, for both $\mathrm{N}_{2} \mathrm{O}$ and $\mathrm{O}_{3}$, scale best in agreement with $D \approx 0.1 \mathrm{~m}^{2} \mathrm{~s}^{-1}$ outside the vortex and with $D \approx 0.001 \mathrm{~m}^{2} \mathrm{~s}^{-1}$ inside. The inside value, which is indeed very small, must be tempered by the small length of the branch inside the vortex during this flight.

The 27 January 2000 flight is, however, the only one to exhibit a significant level of tracer fluctuations inside the polar vortex. All the 8 flights done entirely within the polar vortex exhibit very few tracer fluctuations when the ER-2 flies on level legs (on a slightly climbing trajectory actually), as if the vortex was very well mixed during this winter except for a few minor intrusions (Jost et al., 2002) or anomalous diabatic events. In fact, strong sudden warmings which are the main source of variability within the vortex did not occur during 2000 Arctic winter before mid-March. As a result, the comparison of observed and reconstructed transects is also a test for the transport errors due to the analysed winds used in the reconstruction. Figure 8 compare observed and reconstructed transects for 7 March 2000. Except near the dip, the observed transects for both $\mathrm{N}_{2} \mathrm{O}$ and $\mathrm{O}_{3}$ do not exhibit any other structures than small-scale fluctuations over the constant level legs. These fluctuations exceed only occasionally the precision for $\mathrm{N}_{2} \mathrm{O}$ but can be considered as significant for $\mathrm{O}_{3}$ which is measured with better accuracy. It is also visible from the $\mathrm{N}_{2} \mathrm{O}$ reconstruction that the reconstruction enhances a number of structures at scales larger than $100 \mathrm{~km}$ (about $8 \mathrm{~min}$ of flight) that are absent from the observations. In particular, a spurious maximum is obtained near 11:00 UT. Through the cascade process of chaotic advection, these structures generate small-scale fluctuations requiring a diffusivity $D \approx 0.1 \mathrm{~m}^{2} \mathrm{~s}^{-1}$ to fit the roughness of the observations. The $\mathrm{O}_{3}$ reconstruction does not exhibit the same amount of spurious mesoscale structures as $\mathrm{N}_{2} \mathrm{O}$ and hence generates less small-scale structures, resulting into a value of $D \approx 0.01 \mathrm{~m}^{2} \mathrm{~s}^{-1}$ fitting observed roughness. The spurious structure near 11:00 UT is, however, seen in the standard deviation. Our interpretation of this discrepancy is that the spu-
Table 1. Table of estimated Lagrangian diffusivity based on roughness curves for the 10 processed flights of the campaign and the return flights of 16 and 18 March. The two flights of 27 January 2000 and 11 March 2000 have been split into legs inside (i) and outside (o) the polar vortex. All the other flights between 20 January and 12 March are inside the polar vortex. The two flights on 16 and 18 March are within the surf zone.

\begin{tabular}{|c|c|c|}
\hline Date & $\begin{array}{l}D \text { from } \\
\mathrm{N}_{2} \mathrm{O}\left(\mathrm{m}^{2} \mathrm{~s}^{-1}\right)\end{array}$ & $\begin{array}{l}D \text { from } \\
\mathrm{O}_{3}\left(\mathrm{~m}^{2} \mathrm{~s}^{-1}\right)\end{array}$ \\
\hline 20 Jan. & $\gtrsim 0.01$ & $\gtrsim 0.01$ \\
\hline 27 Jan. i & $\gtrsim 0.001$ & $\gtrsim 0.01$ \\
\hline 27 Jan. o & 刃 0.1 & 刃 0.1 \\
\hline 31 Jan. & $\approx 0.1$ & $\approx 0.01$ \\
\hline 02 Feb. & $\lesssim 0.1$ & $\approx 0.01$ \\
\hline 03 Feb. & $\approx 0.01$ & $\gtrsim 0.01$ \\
\hline 26 Feb. & $\lesssim 0.1$ & $\stackrel{\widetilde{\approx}}{\approx} 0.01$ \\
\hline 05 March & $\approx 0.1$ & $\approx 0.01$ \\
\hline 07 March & $\approx 0.1$ & $\approx 0.01$ \\
\hline 11 March i & $\approx 0.01$ & $\approx 0.001$ \\
\hline 11 March o & $\approx 0.01$ & $\approx 0.05$ \\
\hline 12 March & $\approx 0.1$ & $\lesssim 0.1$ \\
\hline 16 March & $\approx 0.1$ & $\approx 0.1$ \\
\hline $18 \mathrm{March}$ & $\approx 0.1$ & $\approx 0.1$ \\
\hline
\end{tabular}

rious structures in the $\mathrm{N}_{2} \mathrm{O}$ reconstruction are due to spurious vertical transport in the vertical $\mathrm{N}_{2} \mathrm{O}$ gradient and that the lower sensitivity of $\mathrm{O}_{3}$ is due to its weaker vertical gradient measured by the height-scale $\left[\mathrm{O}_{3}\right] \partial p / \partial\left[\mathrm{O}_{3}\right] \approx 128 \mathrm{hPa}$ compared to $56 \mathrm{hPa}$ for $\mathrm{N}_{2} \mathrm{O}$ at $60 \mathrm{hPa}$ inside the polar vortex in early March. It is worth noticing that the reconstruction tends to generate less spurious structures than REPROBUS itself; this result might be due to the fact that REPROBUS uses 6-hourly analysed winds while our reconstructions are based on 3-hourly winds (see Sect. 9 below). There are also indications that we are able to reconstruct the small maximum denoted as E in Fig. 8, just before the dip, which has been identified as an intrusion of mid-latitude air by Jost et al. (2002) and Konopka et al. (2004).

Table 1 summarizes the estimates of $D$ based on roughness for $\mathrm{N}_{2} \mathrm{O}$ and $\mathrm{O}_{3}$, and checked by visual inspection, for all the flights of the SOLVE campaign from Kiruna. It is apparent that previous results are mostly confirmed during the whole campaign with the noticeable exception of 11 March 2000 flight during which fairly small diffusion was observed outside the polar vortex in the $\mathrm{N}_{2} \mathrm{O}$ reconstructions.

Finally, Fig. 9 compares observed and reconstructed transects for the return flight of 16 March 2000 from Kiruna to North America, that occurred entirely outside the vortex and confirms the estimate $D \approx 0.1 \mathrm{~m}^{2} \mathrm{~s}^{-1}$ in the surf zone. 

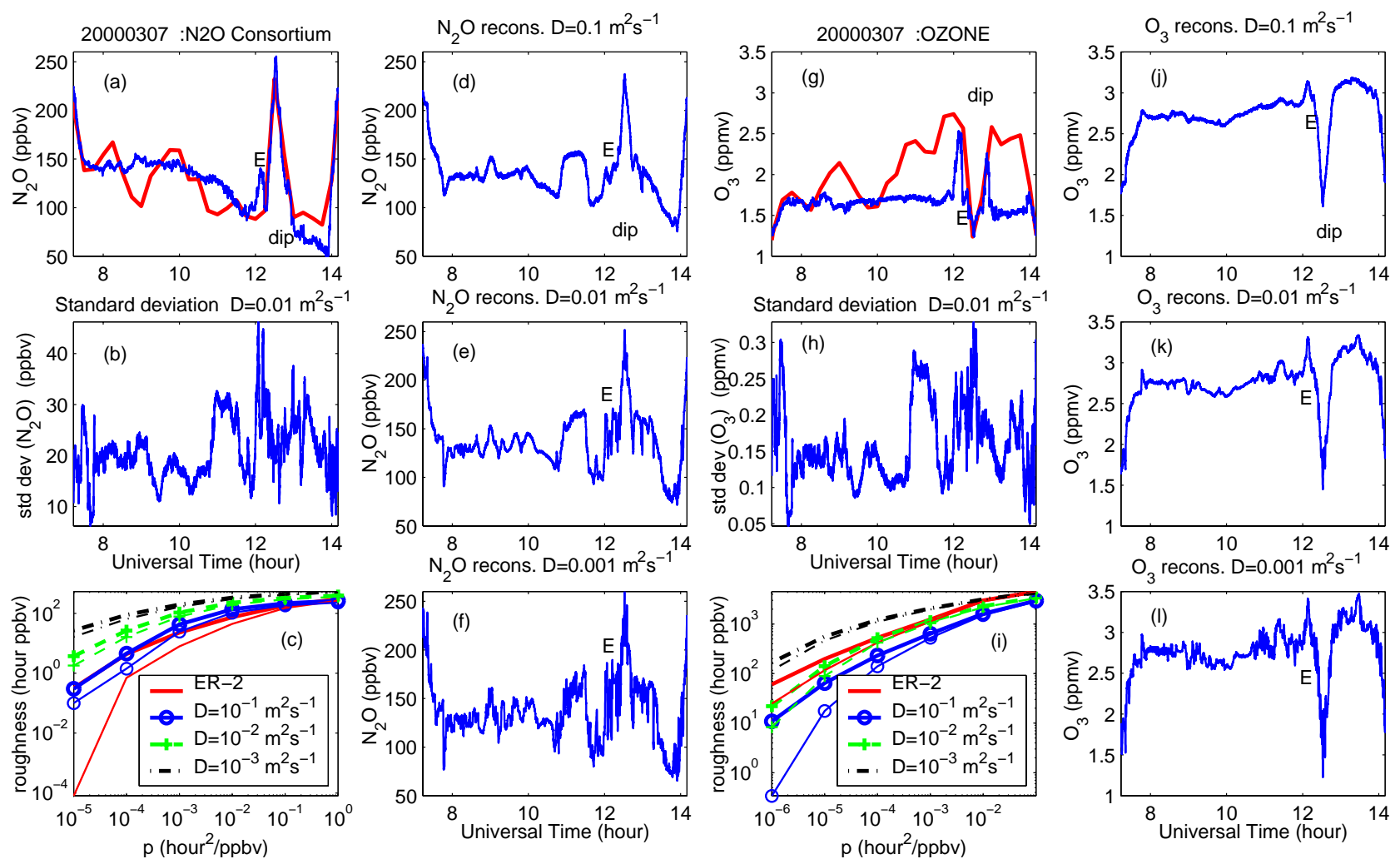

Fig. 8. Reconstructions and roughness for the 07 March 2000 flight inside the polar vortex. Left six panels for $\mathrm{N}_{2} \mathrm{O}$ and right six panels for $\mathrm{O}_{3}$. (a): Observed (blue) and REPROBUS (red) $\mathrm{N}_{2} \mathrm{O}$. (d, e, f): $\mathrm{N}_{2} \mathrm{O}$ reconstructions for $D=0.1,0.01$ and $0.001 \mathrm{~m}^{2} \mathrm{~s}^{-1}$ at $\tau=34$ days. (b): Standard deviation of $\mathrm{N}_{2} \mathrm{O}$ among the $N$ particles released from each location. (c): Roughness for observed and reconstructed transects as indicated in the legend. Thick lines: shift is $\sigma=3$ ppbv; thin lines: shift is $2 \sigma$. (g-l): Same as (a-h) but for $\mathrm{O}_{3}$ and $\sigma=10$ ppbv.

\section{Local variations of turbulent diffusion}

As already noticed in Section 4, the 11 March 2000 flight crossed a sheet of polar air at some distance outside the vortex edge. This sheet is the remain of a fairly broad streamer emitted from the vortex by 28 February 2000, 13 days earlier. On 11 March, its signature was very faint in the ECMWF analysed potential vorticity (not shown) but it was still very well preserved in the $\mathrm{N}_{2} \mathrm{O}$ field. Its weak signature in the $\mathrm{O}_{3}$ field is due to the weak horizontal $\mathrm{O}_{3}$ gradient in the region where it originates from.

Figure 10 shows an enlargement of the sheet crossing for the observed and reconstructed transects. The flight was mainly along mean tracer gradients and the observed sheet was met $325 \mathrm{~km}$ after crossing the vortex edge. The sheet is $120 \mathrm{~km}$ large and the striking feature is the asymmetry of its two edges. The south edge is smooth and fits very well an error function with width $32 \mathrm{~km}$. The north edge is steep with a width of about $2.5 \mathrm{~km}$. The reconstruction succeeds in reproducing the sheet albeit it is slightly displaced southward by $104 \mathrm{~km}$ but the asymmetry of the sheet cannot be reproduced with a single value of the diffusivity which generates the same slope on both edges. The observed south slope of $1.49 \mathrm{ppbv} \mathrm{km}^{-1}$ lies between that for $D=0.1$ and
$0.01 \mathrm{~m}^{2} \mathrm{~s}^{-1}$, respectively 1.22 and $2.64 \mathrm{ppbv} \mathrm{km}^{-1}$, closer to the first one. The observed north slope is as steep as that for $D=0.001 \mathrm{~m}^{2} \mathrm{~s}^{-1}$. Hence, a variation of more than one order of magnitude for the Lagrangian turbulent diffusion occurs over a short distance of about $100 \mathrm{~km}$ across the sheet.

It would not be surprising to observe such variations in the instantaneous turbulent diffusion which is expected to be very intermittent in time and space, but the persistence of sharp variations in the averaged Lagrangian diffusivity indicates the presence of a transport barrier. Noting that large fluctuations, compatible with low diffusion, are observed between the filament and the vortex edge, it is tempting to suggest that the dynamical vortex edge does not coincide in this case with the chemical edge but is located right in the center of the sheet.

\section{Relation between diffusion and dispersion}

A convenient way to parametrize turbulent diffusion is to assume a relation between dispersion and turbulent diffusion as initially done in Prandtl's mixing length hypothesis and applied since then in more refined models like Smagorinsky parametrization (see, e.g., Pope (2000)). In the transport model CLAMS (McKenna et al., 2002; Konopka et al., 


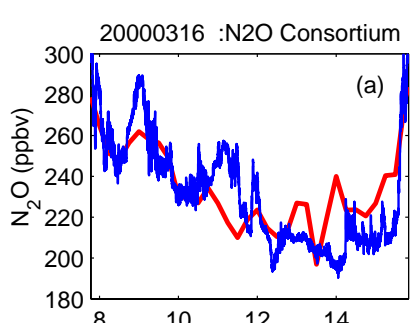

Standard deviation $D=0.01 \mathrm{~m}^{2} \mathrm{~s}^{-1}$
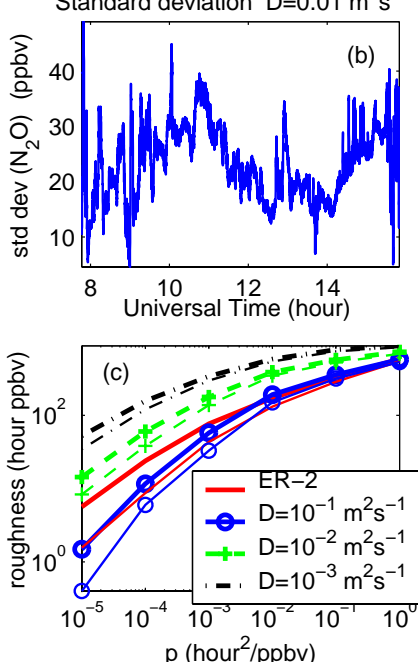
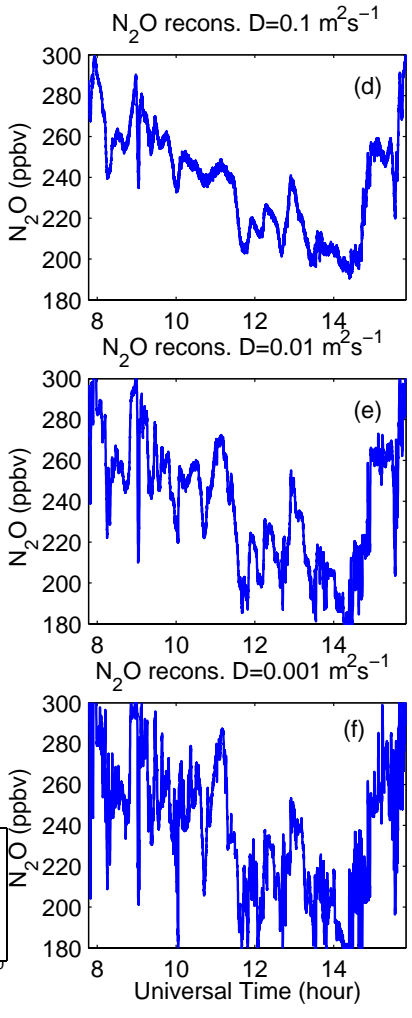
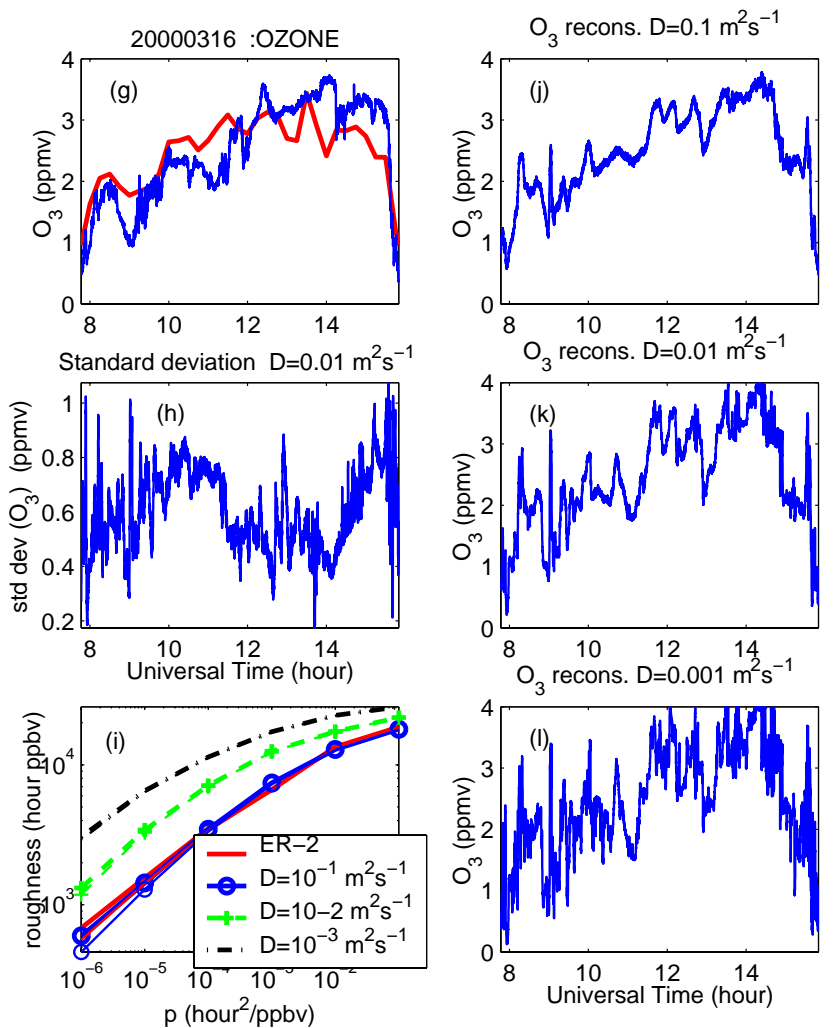

Fig. 9. Same as Fig. 8 but for the 16 March 2000 flight outside the polar vortex and $\tau=29$ days.
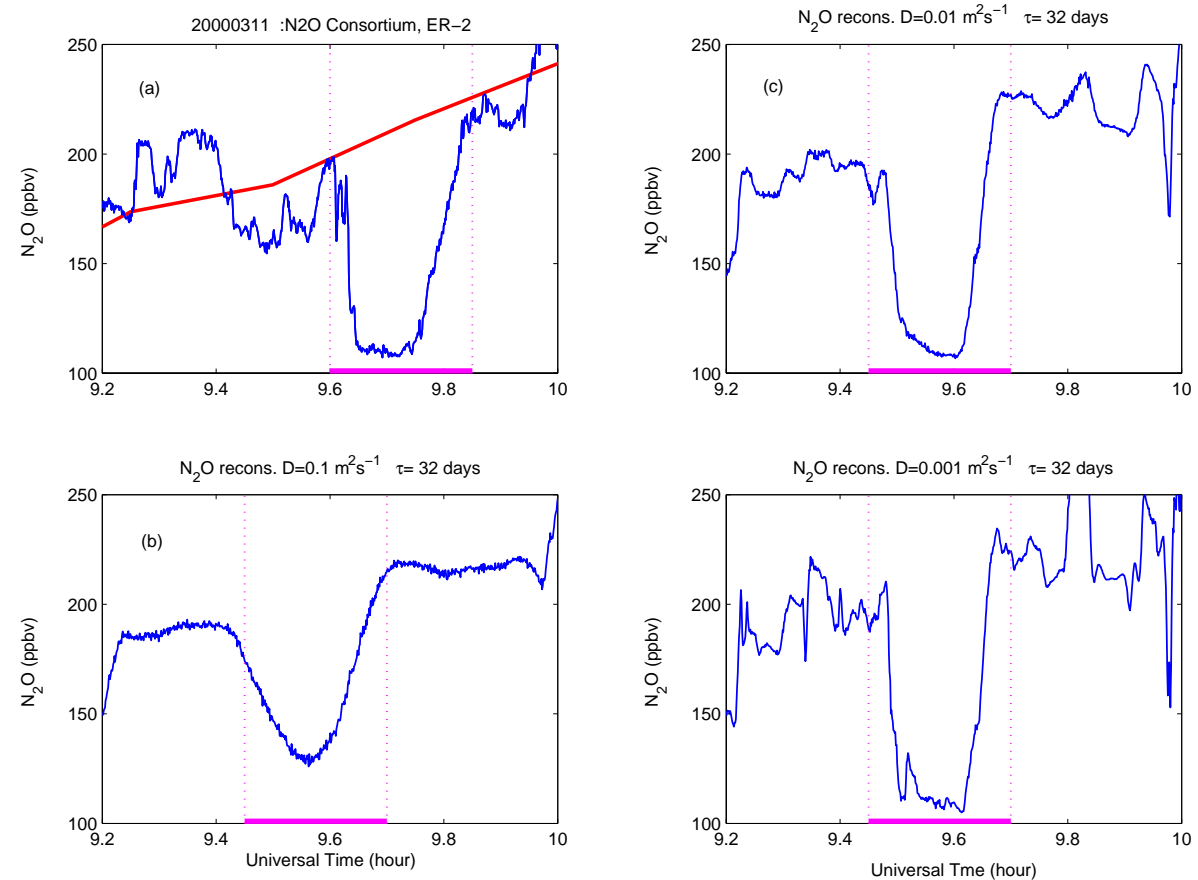

Fig. 10. Enlargement of observed and reconstructed transects encompassing the first crossing of the polar air sheet during 11 March 2000 flight. (a): ER-2 (blue) and REPROBUS (red) $\mathrm{N}_{2} \mathrm{O}$. (b-d): Reconstructed $\mathrm{N}_{2} \mathrm{O}$ for $D=0.1,0.01$ and $0.001 \mathrm{~m}^{2} \mathrm{~s}^{-1}$ for $\tau=32$ days. 


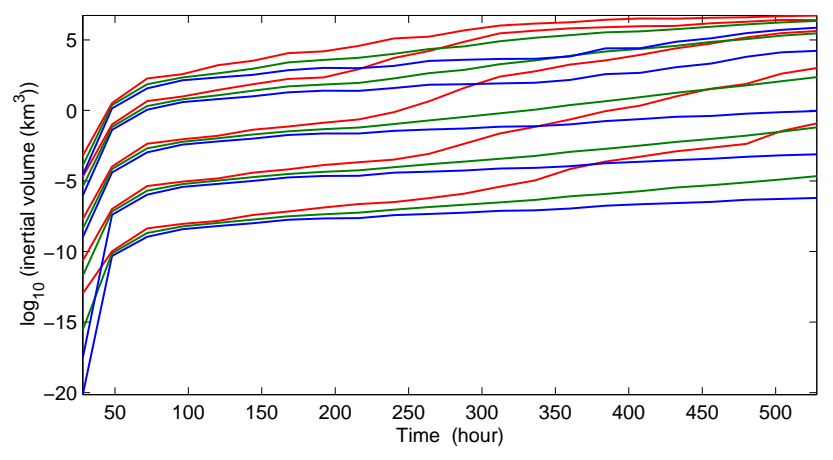

Fig. 11. Inertial volume calculated for the cloud of $N$ particles emitted from the flight track every 4 seconds. The inertial volume is calculated by diagonalizing the inertia matrix of the cloud and taking the square-root of the product of the diagonal terms. Families of curves from bottom to top: $D=10^{-8} \mathrm{~m}^{2} \mathrm{~s}^{-1} \quad D=10^{-6} \mathrm{~m}^{2} \mathrm{~s}^{-1}$ $D=10^{-4} \mathrm{~m}^{2} \mathrm{~s}^{-1} \quad D=10^{-2} \mathrm{~m}^{2} \mathrm{~s}^{-1} \quad D=10^{-1} \mathrm{~m}^{2} \mathrm{~s}^{-1}$. Green: average $\log$ (volume); blue: $5 \%$ percentile of the distribution; red: $95 \%$ of the distribution. The distribution consists in 500 points over a selected $2000 \mathrm{~s}$ section of the 27 January 2000 flight.

2004), mixing is parametrized as a function of the deformation of a grid of points advected by the flow.

Since turbulent diffusion is estimated here independently of any relation with dispersion, it is interesting to compare both quantities.

\subsection{Onset of dispersion}

First we check the consistency of our numerical calculations with respect to the dynamics of advection and diffusion. Starting from a spatial $\delta$-distribution, diffusion initially dominates and the inertial axis of the cloud of $N$ particles grow as $D^{1 / 2} t^{1 / 2}$. This applies here to all axes even if diffusion only acts in the vertical direction because the time step $\delta t$ is such that $\Lambda \delta t=O(1)$. This first stage ends when the cloud reaches a size $\ell_{d} \approx D^{1 / 2} \gamma^{-1 / 2}$ for which diffusion equilibrates with strain in one direction, after which expansion of the cloud pursues exponentially with a rate of the order $\gamma$ as a pancake or a filament (depending on the number of unstable directions) while the smaller transverse dispersion remains of the order of $\ell_{d}$.

The duration $t^{*}$ of the first stage satisfies $D^{1 / 2} t^{* 1 / 2} \sim$ $D^{1 / 2} \gamma^{-1 / 2}$ and hence should be independent of $D$. This is checked in Fig. 11 showing the growth of the inertial volume for a wide range of diffusivity values, including unrealistic sub-molecular values. The diffusive stage for all cases ends at the same $t^{*} \approx 3$ days. The subsequent growth rate of the inertial volume is bounded by two curves showing the 5\% and 95\% percentiles of the distribution. The growth is exponential as long as the largest size of the cloud remains small with respect to the characteristic scale of strain variation and the cloud retains an ellipsoidal shape. This linearity condition is more easily satisfied for the 5\% percentile which corresponds to a growth rate of 0.26 days $^{-1}$. The upper $95 \%$ percentile grows initially at a higher rate of 0.63 days $^{-1}$. For the two largest diffusions tested here, the size of the cloud rapidly violates the linearity condition and the growth weakens as the cloud distorts and mixes with itself.

\subsection{Lyapunov exponents}

A geometric measure of deformation induced by strain is provided by the Lyapunov exponents (Pierrehumbert and Yang, 1993). For non diffusive motion, they describe the transformation of an infinitesimal spherical cloud surrounding a particle at time $t_{0}$ into an ellipsoid at time $t_{1}$ in a local reference frame relative to the particle. If $\delta \boldsymbol{x}(t)$ is an infinitesimal deviation at time $t$, its evolution is described by the tangent linear operator $\mathbf{M}$ as $\delta \boldsymbol{x}\left(t_{1}\right)=\mathbf{M}\left(t_{0}, t_{1}\right) \delta \boldsymbol{x}\left(t_{0}\right)$ and the local finite-time Lyapunov exponents $\lambda_{i}$ are related to the eigenvalues $\sigma_{i}$ of $\mathbf{M}^{t} \mathbf{M}$ by $2 \lambda_{i}=1 /\left(t_{1}-t_{0}\right) \ln \sigma_{i}$ where superscript $t$ denotes transposition. A convenient way to calculate the local Lyapunov exponents is by finite difference using a small initial perturbation in three orthogonal directions and performing, at regular intervals, an estimate of the growth of length, surface and volume, followed by an orthonormalization procedure that regenerates an initial trihedron for the following interval (for more details, see Benettin et al. (1980); Ott (1993)). It can be shown (Goldhirsch et al., 1987) that, after a transient time, this method provides the three local Lyapunov exponents $\lambda_{1} \geqq \lambda_{2} \geqq \lambda_{3}$. Since $\mathbf{M}$ can be calculated as a by-product of the procedure for short times, it has been checked that this is true for $t_{1}-t_{0}>20$ days. The norm used in the orthogonalization takes into account the aspect ratio of stratospheric structures by magnifying the vertical direction by a factor $\alpha$ with respect to the horizontal directions. Doing so, we define a distance in terms of tracer difference rather than in terms of metric separation. Although infinite time Lyapunov exponents are independent on the norm, finite time exponents and convergence to the large time limit depend on it. The Lyapunov exponents are calculated over the duration $\tau$ (that is $\tau=t_{1}-t_{0}$ ) near the single trajectory obtained for $D=0$ and the orthogonalization interval is one day.

For backward evolution in time, the smallest exponent $\lambda_{3}$ describes the exponential elongation of line segments, while $\lambda_{1}+\lambda_{2}$ describes the growth rate of surface elements and $\lambda_{1}+\lambda_{2}+\lambda_{3}$ describes the growth rate of the volume. Since the flow is close to incompressible, the sum of the three exponents should be close to zero. Three cases can hold:

1. $\lambda_{1} \approx \lambda_{2}>0, \lambda_{3} \approx-\lambda_{1}-\lambda_{2}$ which means that sheets are formed if $t>0$ and filaments if $\tau<0$.

2. $\lambda_{1}>0$ and $\lambda_{2} \approx \lambda_{3}<0$ which means that sheets are formed if $t<0$ and filaments if $\tau>0$

3. $\lambda_{1}>0, \lambda_{2} \approx 0$ and $\lambda_{3} \approx-\lambda_{1}<0$ which means that filaments are formed forward and backward in time. 

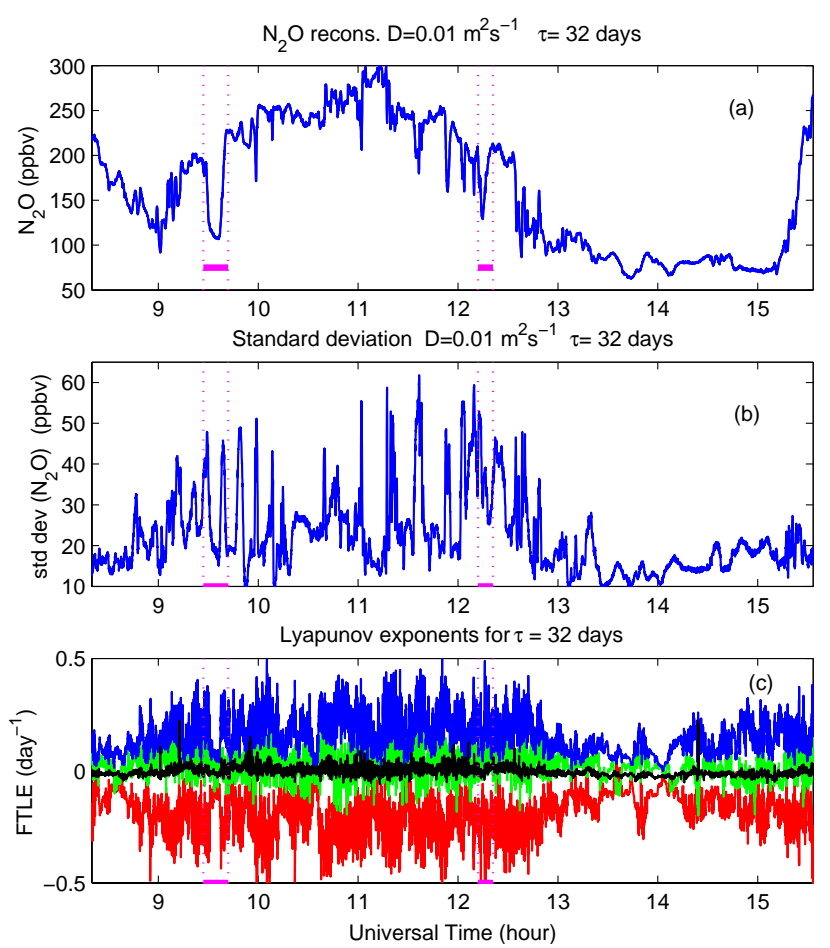

Fig. 12. (a) $\mathrm{N}_{2} \mathrm{O}$ reconstruction for the 11 March 2000 flight with $D=0.01 \mathrm{~m}^{2} \mathrm{~s}^{-1}$ and $\tau=32$ days. (b) $\mathrm{N}_{2} \mathrm{O}$ standard deviation of the $N$ particles within each diffusive ensemble. (c) Lyapunov exponents, blue: $\lambda_{1}$, green: $\lambda_{2}$, red: $\lambda_{3}$, black: $\lambda_{1}+\lambda_{2}+\lambda_{3}$. The location of the polar air sheet crossings is outlined.

For diffusive motion and backward in time, the negative exponents describe the elongation of the cloud of particles emitted from each point for $|\tau|>t^{*}$.

Figure 12 shows the $\mathrm{N}_{2} \mathrm{O}$ reconstruction and Lyapunov exponent for 11 March 2000 and $\tau=32$ days. Since the calculation is based on single deterministic trajectories, the Lyapunov exponents are noisy but several properties can be drawn from the figure. There is a clear separation in magnitude between the inside of the polar vortex, with typical values smaller than 0.1 day $^{-1}$ and small tracer standard deviation, and the outside, with typical values of the order of 0.25 day $^{-1}$ and large tracer standard deviation. Hence, the inside of the polar vortex is much less strained than the outside. This is in agreement with previous studies of the winter polar stratosphere (Pierce and Fairlie, 1993; Bowman, 1993). Larger dispersion, especially in the vertical, means sampling a wider range of $\mathrm{N}_{2} \mathrm{O}$ values, and the tracer standard deviation is also larger outside the polar vortex than inside. The unfiltered curves of the most negative Lyapunov exponent and the standard deviation are anti-correlated with a coefficient -0.407 which is significant since it is calculated over 8844 points along the flight. The sum of the three Lyapunov exponents is close to zero. In principle, its variation should correlate with the variation of parcel density which can be
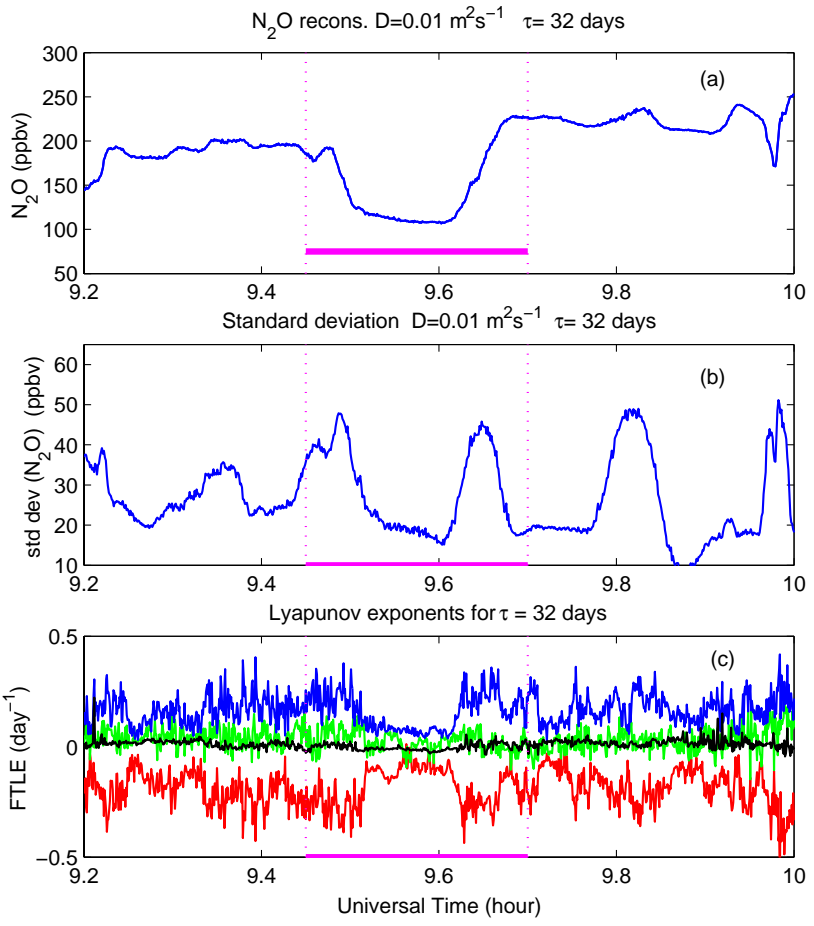

Fig. 13. Enlargement of Fig. 12 encompassing the first crossing of the polar air sheet.

calculated, but we reach here the limit of our numerics and the correlation is very poor. The sign of the intermediate Lyapunov exponent oscillates around 0 along the flight but its magnitude is always much smaller than the two large negative and positive exponents which almost compensate. Hence, we are essentially in the third case defined above.

Figure 13 shows an enlargement around the sheet of polar air. The small dispersion that characterizes the polar air has been preserved inside the sheet in agreement with its isolation from surrounding air. Both edges exhibit a maximum, indicating that strong shear, presumably due to PV jump, has been experienced by the parcels over the last three weeks but there is no distinction between the smooth and the sharp edge. This suggests that the event that led to the smooth south edge is not due to shear induced turbulence but rather to an exterior event such as breaking gravity waves, not unlikely to occur along the cost of Scandinavia.

\section{Sensitivity to temporal resolution}

Our reconstructions are based on using 3-hourly winds obtained by interleaving first guesses with analysis. First guesses for 3-h evolution are archived at ECMWF for the four daily assimilation cycles of the operational model until September 2000. 4-D variational analysis can be used for more recent dates and 3-h and 9-h forecasts can be used with the re-analysis. This is the standard procedure 

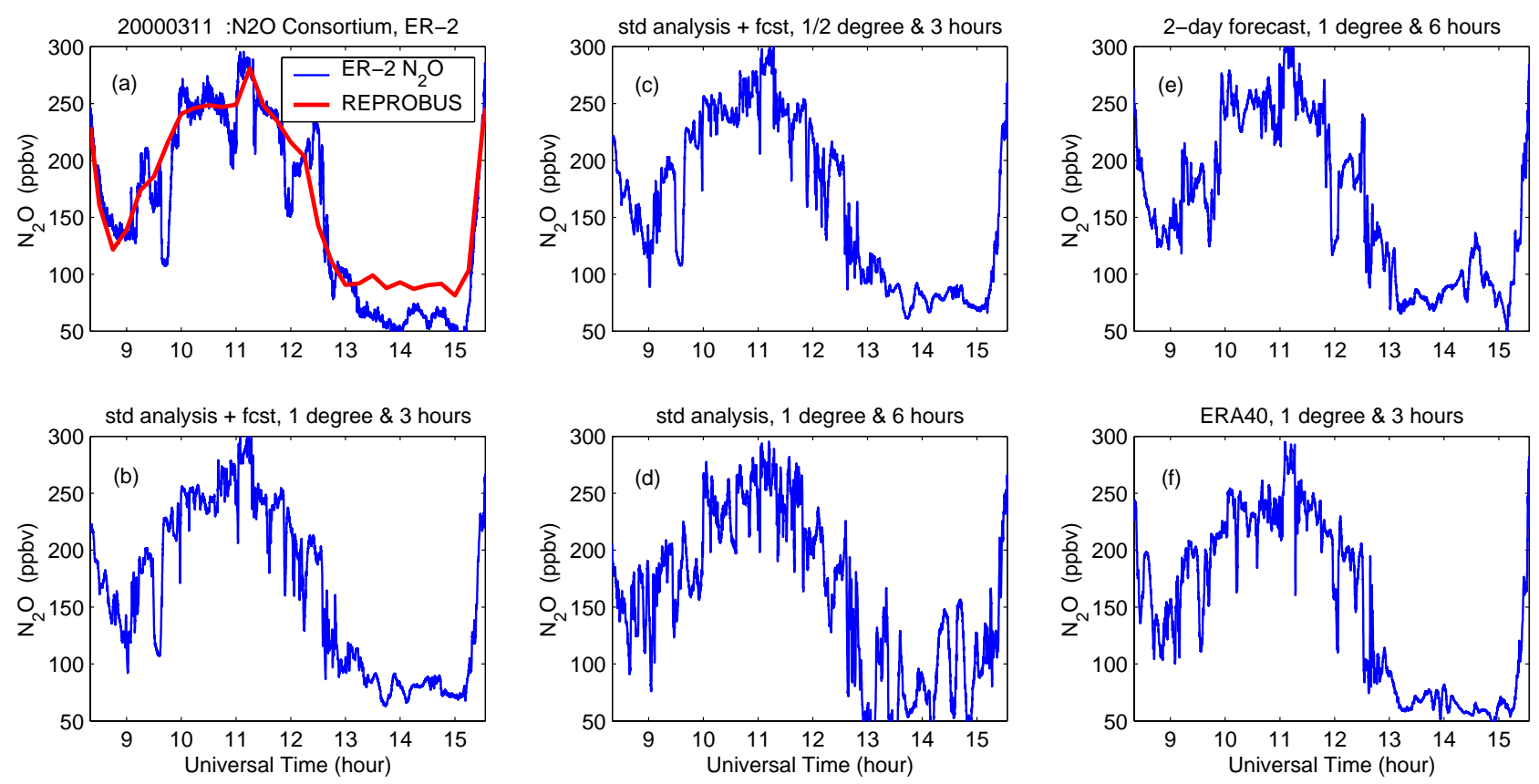

Fig. 14. Comparison of observed $\mathrm{N}_{2} \mathrm{O}$ and reconstructions for different resolutions of the advecting wind. (a) Observed $\mathrm{N}_{2} \mathrm{O}$ and REPROBUS prediction. (b): Reconstructed $\mathrm{N}_{2} \mathrm{O}$ using 3-hourly analysed and first guess winds on a $1^{\circ}$ latitude-longitude grid which is the standard setting for all other figures. (c): Same as (b) but using winds on a $0.5^{\circ}$ grid. (d): Same as (b) but using only the 6-hourly analysed winds. (e): Same as (d) but replacing the analysed winds by 6-hourly forecast winds generated twice-daily from the 48 and 54 hours forecasts. (f): Same as (b) but using the ERA-40 reanalysis instead of the operational analysis. All reconstructions are done with $D=0.01 \mathrm{~m}^{2} \mathrm{~s}^{-1}$ and $\tau=32$ days.

implemented in FLEXPART (Stohl et al., 2002) and inherited in TRACZILLA. A number of other Lagrangian studies in the literature have used instead 6-hourly winds based solely on analysis. The main reason for this choice seems practical since the ECMWF 6-hourly analysis or re-analysis are mirrored and easily available from many locations. It is, however, questionable that the 6-hour archiving period, which has been chosen to provide accurate climatology, is optimum to perform off-line transport calculations. This led us to investigate the effect of changing the temporal resolution of advecting winds onto the reconstruction and estimated turbulent diffusion. An other motivation was a recent work (Stohl et al., 2004) showing that forecasted winds are much less diffusive than analysis. Hence, we have also tested the effect of replacing analysed winds by forecasted winds.

We have performed a series of reconstructions for the same case, namely that of 11 March 2000 which is already well documented in this study, and for a single value of diffusivity $D=0.01 \mathrm{~m}^{2} \mathrm{~s}^{-1}$ by varying the field of advecting winds. The reference reconstruction, shown in Fig. 14b is that performed with 3-hourly winds, one-degree horizontal resolution and 60 hybrid levels in the vertical. Figure $14 \mathrm{c}$ shows the reconstruction obtained by halving the horizontal resolution of advecting winds to $0.5^{\circ}$. The reconstruction is strikingly insensitive to this spatial refinement in agreement with previous observations (Waugh and Plumb, 1994; Methven and
Hoskins, 1999) and the fact that ECMWF analysed winds bear little variance at such small scales in the lower stratosphere. Notice also that near the pole the $1^{\circ}$ longitude-latitude grid already over-resolves the longitudinal variations. Figure $14 \mathrm{~d}$ shows the reconstruction obtained by calculating transport from interpolated 6-hourly analysed winds, dropping the first guesses out of our procedure. The effect is this time dramatic with a strong enhancement of the fluctuations inside and outside the polar vortex. The sheet is still visible but can easily be confounded with other spurious structures. In order to dwell into the source of this considerable deterioration, we have then performed a reconstruction based on forecasted wind, still at 6-h interval, by using the 48-h and 54-h forecasts from the deterministic forecast runs performed twice-daily at ECMWF. Figure $14 \mathrm{e}$ is constructed from this new 6-hourly dataset. With respect to Fig. 14c, the reconstruction shows a considerable damping of the fluctuations and much better agreement with the observations. We could not test the reconstruction performed with 3-hourly forecasts since they have not been archived at ECMWF before 2002. Finally, Fig. 14f shows the reconstruction obtained from 3hourly winds taken from the ERA40 re-analysis instead of the operational analysis. The reconstruction differs from the reference but rivals in skill compared to the observation with the noticeable exception of the polar air sheet which is badly predicted. 
These results indicate that off-line transport calculations are highly sensitive to the lack of resolution of the wind fluctuations in 6-hourly analysis. Fluctuations are mostly seen in the vertical wind calculated from the divergence field, notoriously noisy in the analysis, using the mass conservation. Whatever the true timescale of a given fluctuation, sampling the wind field every $x$ hours makes it persistent over $x$ hours. As a result, vertical displacements are overestimated and in turn, eddy transport along vertical tracer gradients is also overestimated. In the limit of very short-lived fluctuations, it can be shown easily that vertical eddy transport is proportional to the sampling interval. For our prospect of measuring turbulent diffusion, the excess transport leads to overestimate Lagrangian diffusion in order to smooth out the excess fluctuations in the reconstructed tracer.

Not only the fluctuations in the 6-hourly winds are undersampled, they are also to a large extend spurious as demonstrated from the comparison between analysed and forecast winds. In spite of the efforts to filter out gravity waves during assimilation, the analysed winds contain a significant level of spurious motion which is damped during the forecast as the model relaxes to its attractor (Kalnay, 2003). This spurious motion presumably consists mainly in short-period gravity waves. Wavy structures with scales of the order of several tens to a few hundreds $\mathrm{km}$ are often seen over the displacements of the cloud of parcels emitted from a single location or even in the reconstruction itself. Figure 15 shows such an example.

However, it is still unclear whether $3 \mathrm{~h}$ (the maximum frequency at which wind datasets are currently available) is a sufficiently short interval to achieve satisfying reconstructions. In fact, the discrepancy between estimates of $D$ from $\mathrm{N}_{2} \mathrm{O}$ and $\mathrm{O}_{3}$ inside the vortex suggests that this is not the case and that our estimate of $D$ is still an upper bound of the true value representing unresolved turbulent motion.

\section{Conclusions}

We have shown that the sensitivity of Lagrangian reconstruction to the integration time of back-trajectories disappears when diffusion is added as a random walk over an ensemble of trajectories and reconstruction is based on statistical average over this ensemble. Using the tracer field produced by REPROBUS as initialisation, it takes 2 to 4 weeks for diffusivity varying from $0.1 \mathrm{~m}^{2} \mathrm{~s}^{-1}$ to $0.001 \mathrm{~m}^{2} \mathrm{~s}^{-1}$ to reach a stage where small-scale structures are essentially invariant under extension of the reconstruction time.

The comparison of ER-2 measurements of $\mathrm{N}_{2} \mathrm{O}$ and $\mathrm{O}_{3}$ with reconstructions performed with varying diffusivity provides an estimate of turbulent diffusivity of the order of $0.1 \mathrm{~m}^{2} \mathrm{~s}^{-1}$ in the surf zone and one order of magnitude less, at least, inside the polar vortex, based on the statistical distribution of tracer fluctuations. When well-defined structures are identified in the observations and the reconstructions, a

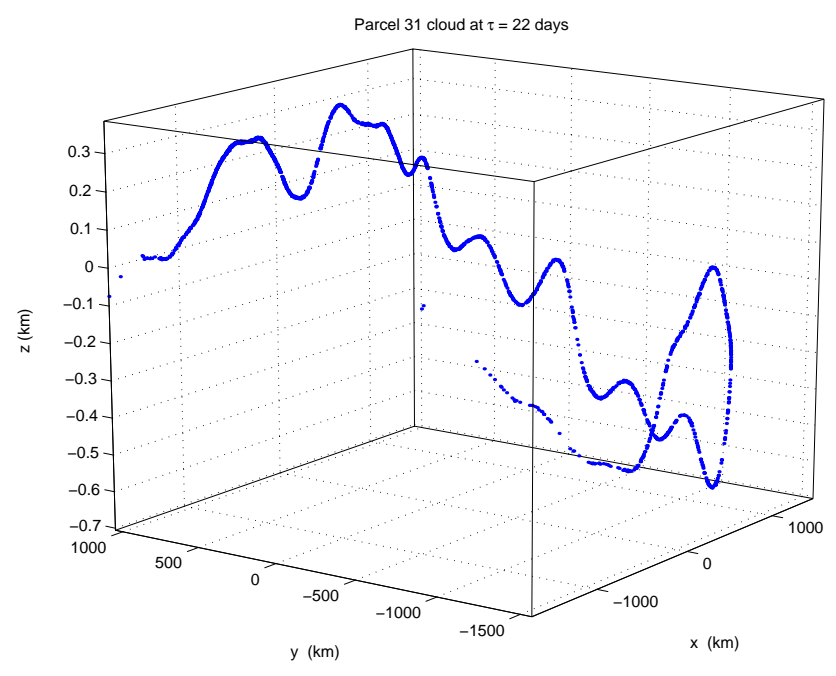

Fig. 15. Distribution of the cloud of points at $\tau=22$ days for a parcel along the 11 March 2000 flight track. Calculation is done with $D=10^{-4} \mathrm{~m}^{2} \mathrm{~s}^{-1}$ in order to reduce lateral dispersion and to magnify the wavy pattern. It can be checked that dispersion occurs as a filament.

local estimate of diffusivity is possible, and large variations of more than one order of magnitude have been observed across the width of a polar vortex sheet.

The dispersion measured by Lyapunov exponent is much reduced inside the polar vortex compared to outside. Therefore, models that parametrize mixing based on deformation, like CLAMS (Konopka et al., 2004) would correctly predict less mixing inside the polar vortex than outside. However, the absence of correlation between diffusion and dispersion on the two edges of the polar air sheet on 11 March 2000 is an indication that such parametrization may miss numerous mesoscale features.

The estimate of diffusivity within the surf zone is in agreement with previous results based on ozone sounding (Legras et al., 2003). The resolution of standard ozone soundings is so coarse that lower diffusivity could not be tested while the ER-2 data are providing a much higher resolution and are much better suited to study the small-scale fluctuations of tracers.

The estimate of diffusivity in the surf zone is, however, larger by one order of magnitude than the value proposed by Balluch and Haynes (1997) and Waugh et al. (1997). In order to bridge the gap between these studies and ours, we have processed several of the cases studied in Balluch and Haynes (1997). The first of these cases is the 4 January 1992 flight of the AASEII campaign, during which $\mathrm{N}_{2} \mathrm{O}$ was measured with the ATLAS instrument (Loewenstein et al., 1990). Reconstruction is here based on winds from the ERA40 re-analysis and a REPROBUS run with low horizontal resolution of $6^{\circ} \times 6^{\circ}$. Figure 16 shows observed and reconstructed $\mathrm{N}_{2} \mathrm{O}$. Clearly, the reconstructed values 

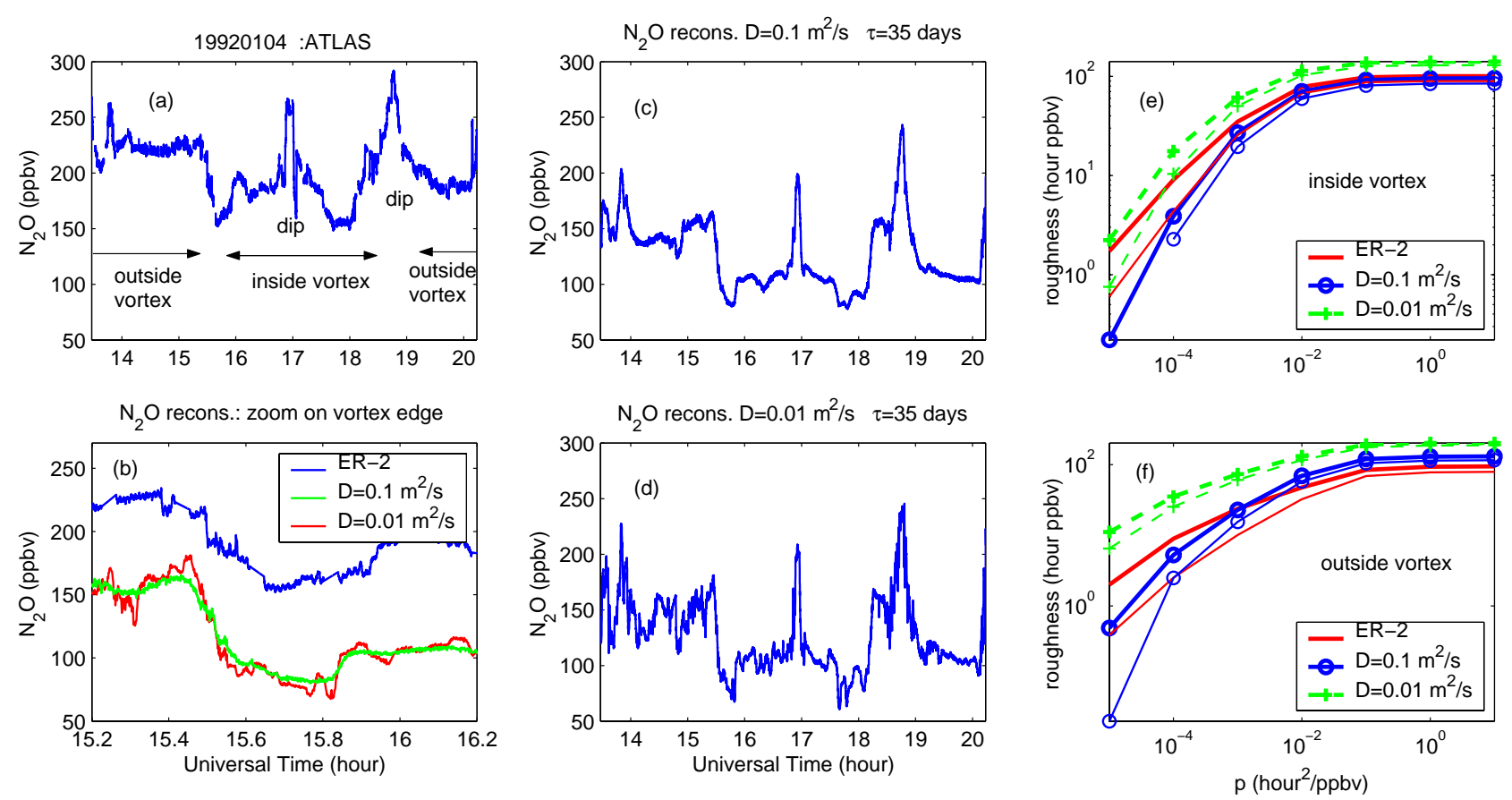

Fig. 16. $\mathrm{N}_{2} \mathrm{O}$ reconstruction for the 4 January 1992 flight during AASEII campaign in the Arctic. (a): Measured $\mathrm{N}_{2} \mathrm{O}$ along the flight track. (b): Enlargement of the observed and reconstructed curves near the vortex edge. (c-d): $\mathrm{N}_{2} \mathrm{O}$ reconstruction with $D=0.1 \mathrm{~m}^{2} \mathrm{~s}^{-1}$ and $0.01 \mathrm{~m}^{2} \mathrm{~s}^{-1}$. (e): Roughness of observed and reconstructed transects for the flight segments inside the polar vortex. (f): Roughness of observed and reconstructed transects for the flight segments outside the polar vortex
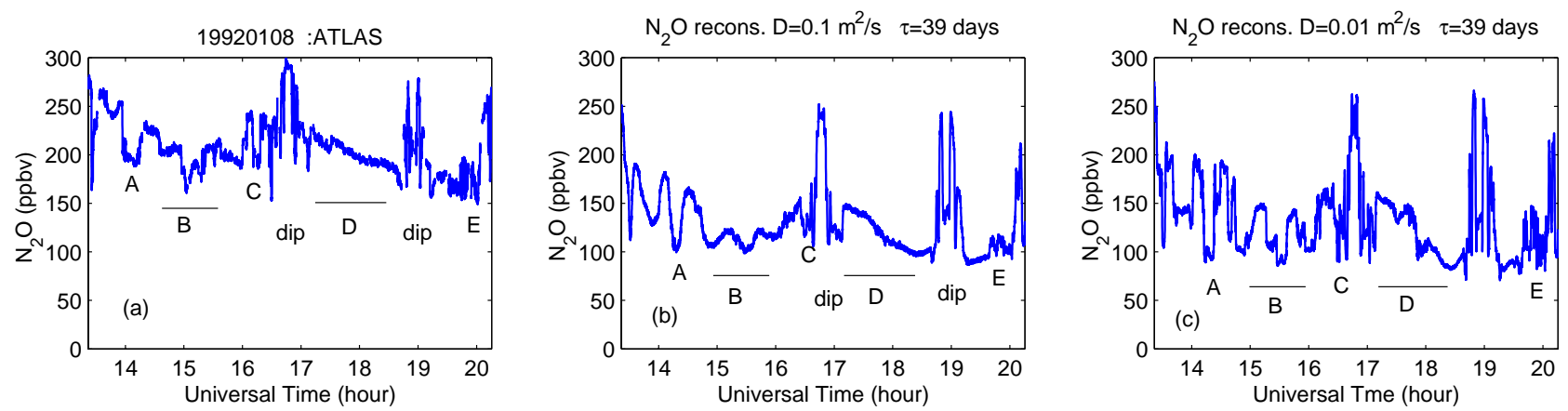

Fig. 17. $\mathrm{N}_{2} \mathrm{O}$ reconstruction for the 8 January 1992 flight during AASEII campaign in the Arctic. (a): Measured $\mathrm{N}_{2} \mathrm{O}$ along the flight track. (b-c): $\mathrm{N}_{2} \mathrm{O}$ reconstruction with $D=0.1 \mathrm{~m}^{2} \mathrm{~s}^{-1}$ and $0.01 \mathrm{~m}^{2} \mathrm{~s}^{-1}$.

are too low by almost $40 \%$ but the variations are fairly well reproduced. Like during SOLVE, there is a large contrast in turbulent diffusion between the inside and the outside of the vortex, with non ambiguous indication of $D \approx 0.1 \mathrm{~m}^{2} \mathrm{~s}^{-1}$ rather than $0.01 \mathrm{~m}^{2} \mathrm{~s}^{-1}$ outside the vortex. Figure $16 \mathrm{~b}$ is an enlargement of the vortex edge corresponding to Fig. 9 of Balluch and Haynes (1997), showing that the observed $\mathrm{N}_{2} \mathrm{O}$ agrees better with our large diffusion rather than with the small value. Among the two other cases studied by Balluch and Haynes (1997), the sheet is entirely missed in our reconstruction of 7 May 1993 flight due to the very short track and Fig. 17 shows the reconstructions of the 8 January 1992 flight. We do not reproduce correctly the sheet shown on Fig. 8 of Balluch and Haynes (1997) (structure C on Fig. 17) but it appears that this flight outside and in the edge of the polar vortex exhibits a complex juxtaposition of small and large diffusion. The broad sheet marked as A in Fig. 17 is another example of contrast between smooth and a sharp edges that can be accounted only by a similar contrast in diffusion. Region B shows a mixture of sharp and smooth jumps that require a mixture of diffusions, while the absence of jumps in region $\mathrm{D}$ is in favor of large uniform diffusion. In region $\mathrm{E}$ again, large fluctuations are compatible with small diffusion. Hence we cannot say at this point that our results disagree 
with Balluch and Haynes (1997) and Waugh et al. (1997). These studies were based on a very small amount of data and it is quite possible that they sampled domains of small diffusion around the polar vortex. The sample of cases outside the polar vortex is also limited in the SOLVE campaign, and further studies are required to establish the spatial and temporal variations of turbulent diffusion in the lower stratosphere.

The reconstructions are found to be sensitive to the quality of the wind fields used for the advection. In particular, the ECMWF analysis seems to contain a significant amount of short-lived fluctuations which induce spurious transport, especially if advecting winds are interpolated between 6-hourly standard ECMWF archived winds.

This spurious effect is probably also affecting transport in REPROBUS. For instance the overestimation of $\mathrm{N}_{2} \mathrm{O}$ within the polar vortex on 11 March 2000 (see Fig. 3) is also found in the CLAMS model when mixing is set at a too high value (Konopka et al., 2004, Fig. 8). Our reconstruction based on 3-hourly winds reduces the spurious mixing and hence decreases the value of $\mathrm{N}_{2} \mathrm{O}$ towards the observed value. The fact that slightly lower values than observed are obtained in Fig. 3(i) could be attributed to errors in the initial REPROBUS field or too strong diabatic descent within the vortex.

The turbulent diffusivity estimated in this study is the combination of the unresolved turbulent motion (the "true turbulent diffusivity") and the diffusion required to filter out the structures induced by the spurious motion contained in the analysed winds. The second effect is likely to hinder the first one as suggested by the comparison of results obtained with $\mathrm{N}_{2} \mathrm{O}$ and $\mathrm{O}_{3}$. It is also possible that chemical reactions contribute to smooth the spatial fluctuations of $\mathrm{O}_{3}$ but it is not easy to conceive a possible mechanism since depletion chemistry does not tend to relax $\mathrm{O}_{3}$ to an equilibrium value and $\mathrm{ClO}$ fluctuations should rather increase the ozone fluctuations (Edouard et al., 1996).

Our results indicate that the state-of-the-art in off-line transport studies, which is mainly based on 6-hourly analysed winds, is far from being satisfactory. A significant improvement is provided by interleaving first guesses to provide 3-hourly winds but it is yet unclear that these winds do not contain significant signature of high-frequency fluctuations as aliases, and hence are still inducing excess transport with respect to what would be found by performing on-line calculations. This latter solution does not seem practical with an operational weather forecast model but archiving winds at higher resolution could be considered, at least for test purpose over a limited period of time. Another possibility would be to archive time-averaged winds instead of instantaneous snapshots. The fact that the high frequency fluctuations are to some large extend a spurious effect of the assimilation could be perhaps circumvented by using winds from short-time forecast, as suggested by the strong improvement shown in Fig. 14e. Finally, a number of models, including CLAMS, are using in the stratosphere vertical winds calcu- lated from the local heating rate and the vertical profile of potential temperature. Although such winds are not providing automatic mass conservation they seem to reconstruct better the observed tracer, at least in Fig.12 of Konopka et al. (2004) for 07 March 2000. These questions will be investigated in further work.

Acknowledgements. We acknowledge D. F. Hurst, H.-J. Jost, E. C. Richards, C. R. Webster, J. W. Elkins, S. M.. Schauffler and E. L. Atlas for provided data from ACATS, ARGUS, ALIAS, WAS and ozone instruments on board the ER-2 during the SOLVE campaign. We acknowledge A. Tuck for useful discussions and advices.

Edited by: P. Haynes

\section{References}

Alisse, J.-R., Haynes, P., Vanneste, J., and Sidi, C.: Quantification of stratospheric mixing from turbulence microstructure measurements, Geophys. Res. Lett., 27, 2621-2624, 2000.

Appenzeller, C., Davies, H., and Norton, W.: Fragmentation of stratospheric intrusions, J. Geophys. Res., 101, 1453-1456, 1996.

Balluch, M. and Haynes, P.: Quantification of lower stratospheric mixing processes using aircraft data, J. Geophys. Res., 102, 23 487-23 504, (97JD00 607), 1997.

Benettin, G., Galgani, L., Giagilli, A., and Strelcyn, J.-M.: Lyapunov characteristic exponents for smooth dynamical systems and for Hamiltonian systems; a method for computing all of them. I: Theory, Meccanica, pp. 9-80, 1980.

Bowman, K. P.: Large-Scale Isentropic Mixing Proprieties of the Antarctic Polar Vortex From Analyzed Winds, J. Geophys. Res., 98, 23,013-23,027, 1993.

Edouard, S., Legras, B., Lefèvre, F., and Eymard, R.: The effect of small-scale inhomogeneities on ozone depletion in the Arctic., Nature, 384, 444-447, 1996.

Falkovich, G., Gawȩdski, K., and Vergassola, M.: Particles and fields in fluid turbulence, Rev. Mod. Phys., 73, 913-975, 2001.

Fukao, S., Yamanaka, M., Ao, N., Hocking, W., Sato, T., Yamamoto, M., Nakamura, T., Tsuda, T., and Kato, S.: Seasonal variability of vertical eddy diffusivity in the middle atmosphere. Three-year observations by the middle and upper atmosphere radar, J. Geophys. Res., 99, 18 973-18 987, 1994.

Goldhirsch, I., Sulem, P.-L., and Orszag, S. A.: Stability and Lyapunov stability of dynamical systems: a differential approach and a numerical method, Physica D, 27, 311-337, 1987.

Haynes, P. and Anglade, J.: The vertical-scale cascade of atmospheric tracers due to large-scale differential advection, J. Atmos. Sci., 54, 1121-1136, 1997.

Haynes, P. and Vanneste, J.: Stratospheric tracer spectra, J. Atmos. Sci., 61, 161-178, 2004.

Holzer, M. and Hall, T.: Transit-time tracer-edge distributions in geophysical flows, J. Atmos. Sci., 57, 3539-3558, 2000.

Hurst, D., Schauffler, S., Greenblatt, J., Jost, H., Herman, R., Elkins, J., Romashkin, P., Atlas, E., Donnelly, S., Podolske, J., Loewenstein, M., Webster, C., Flesch, G., and Scott, D.: Construction of a unified, high-resolution nitrous oxide data set for ER-2 flights during SOLVE, J. Geophys. Res., 107(D20), 8271, doi:10.1029/2001JD000 417, 2002. 
Issartel, J. and Baverel, J.: Inverse transport for the verification of the Comprehensive Nuclea Test Ban Treaty, Atmos. Chem. Phys., 3, 475-486, 2003,

\section{SRef-ID: 1680-7324/acp/2003-3-475.}

Jost, H.-J., Loewenstein, M., Greenblatt, J., Podolske, J., Paul Bui, T., Hurst, D., Elkins, J., Herman, R., Webster, C., Schauffler, S., Atlas, E., Webster, C., Schauffler, S., Atlas, E., Newman, P., Lait, L., and Wofsy, S.: Mixing events revealed by anomalous tracer relationships in the Arctic vortex during winter 1999/2000, J. Geophys. Res., 107(D24), 4795, doi:10.1029/2002JD002 380, 2002.

Kalnay, E.: Atmospheric modeling, data assimilation and predictability, Cambridge Univ. Press, Cambridge, UK, 2003.

Konopka, P., Steinhorst, H.-M., Grooß, J.-U., Günther, G., Müller, R., Elkins, J., Jost, H.-J., Richard, E., Schmidt, U., Toon, G., and McKenna, D.: Mixing and ozone loss in the 1999-2000 Arctic vortex: simulations with the three-dimensional Chemical Lagrangian Model of the Stratosphere (CLaMS), J. Geophys. Res., 109(D2), 2315, doi:10.1029/2003JD003 792, 2004.

Lefèvre, F., Brasseur, G. P., Folkins, I., Smith, A. K., and Simon, P.: Chemistry of the 1991-1992 stratospheric winter: Threedimensional model simulations, J. Geophys. Res., 99, 81838195, 1994.

Lefèvre, F., Figarol, F., Carslaw, K., and Peter, T.: The 1997 Arctic ozone depletion quantified from three-dimensional model simulations, Geophys. Res. Lett., 25, 2425-2428, 1998.

Legras, B., Joseph, B., and Lefèvre, F.: Vertical diffusivity in the lower stratosphere from Lagrangian back-trajectory reconstructions of ozone profiles, J. Geophys. Res., 108, 4562, doi:10.1029/2002JD003 045, 2003.

Loewenstein, M., Podolske, J., and Strahan, S.: ATLAS instrument chracterization: accuracy of the AASE and AAOE nitrous oxide datasets, Geophys. Res. Lett., 17, 481-484, 1990.

Lucet, Y.: Faster than the Fast Legendre Transform, the Linear-time Legendre Transform, Numer. Algorithms, pp. 171-185, 1997.

Mariotti, A., Moustaoui, M., Legras, B., and Teitelbaum, H.: Comparison between vertical ozone soundings and reconstructed potential vorticity maps by contour advection with surgery, J. Geophys. Res., 102, 6131-6142, 1997.

Mariotti, A., Mechoso, C., Legras, B., and Chi, Y.: The evolution of the ozone "collar" in the Antarctic lower stratosphere during early August 1994, J. Atmos. Sci., 57, 402-414, 2000.

McKenna, D., Konopka, P., Groo $\beta$, J.-U., Günther, G., Müller, R., Spang, R., Offermann, D., and Orsolini, Y.: A new Chemical Lagrangian Model of the Stratosphere (CLaMS): 1. Formulation of advection and mixing, J. Geophys. Res., 107(D16), 4309, doi:10.029/2000JD000 114, 2002.

Methven, J. and Hoskins, B.: The advection of high-resolution tracers by low-resolution winds, J. Atmos. Sci., 56, 3262-3285, 1999.

Morse, P. and Feschbach, H.: Methods of theoretical physics, McGraw-Hill, 1953.

Nastrom, G. and Eaton, F.: Turbulent eddy dissipation rates from radar observations at 5-20 km at White Sand missile range: New Mexico, J. Geophys. Res., 102, 19 495-19505, 1997.
Orsolini, Y., Hansen, G., Manney, G., Livesey, N., and Hoppe, U.P.: Lagrangian reconstruction of ozone column and profile at the Arctic Lidar Observatory for Middle Atmosphere Research (ALOMAR) throughout the winter and spring of 1997-1998, J. Geophys. Res., 106, 10 011-10 021, 2001.

Ott, E.: Chaos in dynamical systems, Cambridge Univ. Press, 1993.

Pierce, R. B. and Fairlie, T. D.: Chaotic advection in the stratosphere: Implications for the dispersal of chemically perturbed air from the polar vortex, J. Geophys. Res., 98D, 18 589-18 595, 1993.

Pierrehumbert, R. and Yang, H.: Global chaotic mixing on isentropic surfaces, J. Atmos. Sci., 50, 2462-2480, 1993.

Pope, S. B.: Turbulent flows, Cambridge University Press, 770pp, 2000.

Proffitt, M., Steinkamp, M., Powell, J., McLaughlin, R., Mills, O., Schmeltekopf, A., Thompson, T., Tuck, A., Tyler, T., and Winkler, R.: In situ ozone measurements within the 1987 Antarctic ozone hole from a high-altitude ER-2 aircraft, J. Geophys. Res., 94, 16 547-16555, 1989.

Rex, M., Salawich, R., and Harris et al., N.: Chemical depletion of Arctic ozone in winter 1999/2000, J. Geophys. Res., 107, 8276, doi:10:1029/2001JD000 533, 2002.

Sparling, L. and Bacmeister, J.: Scale dependence of tracer microstructure: PDFs, intermittency and the dissipation scale, Geophys. Res. Lett., 28, 2823-2826, 2001.

Stohl, A., Eckhardt, S., Forster, C., James, P., Spichtinger, N., and Seibert, P.: A replacement of simple back trajectory calculations in the interpretation of atmospheric trace substance measurements, Atmos. Environn., 36, 4635-4648, 2002.

Stohl, A., Cooper, O., and James, P.: A cautionary note on the use of meteorological analysis fields for quantifying atmospheric mixing, J. Atmos. Sci., 61, 1446-1453, 2004.

Sutton, R., Maclean, H., Swinbank, R., O'Neill, A., and Taylor, F.: High-resolution stratospheric tracer fields estimated from satellite observations using Lagrangian trajectory calculations, J. Atmos. Sci., 51, 2995-3005, 1994.

Vanneste, J. and Haynes, P.: Intermittent mixing in strongly stratified fluids as a random walk, J. Fluid Mech., 411, 162-185, 2000.

Waugh, D. and Plumb, R.: Contour advection with surgery: A technique for investigating fine scale structure in tracer transport, J. Atmos. Sci., 51, 530-540, 1994.

Waugh, D., Plumb, R., Atkinson, R., Schoeberl, M., Lait, L., Newman, P., Lowenstein, M., Toohey, D., Avallone, L., Webster, C., and May, R.: Transport of material out of the stratospheric Arctic vortex by Rossby wave breaking, J. Geophys. Res., 99, 10711088, 1994.

Waugh, D., Plumb, R., Elkins, J., Fahey, D., Boering, K., Dutton, G., Volk, C., Keim, E., Gao, R.-S., Daube, B., Wofsy, S., Lowenstein, M., Podolske, J., Chan, K., Proffitt, M., Kelly, K., Newman, P., and Lait, L.: Mixing of polar air into middle latitudes as revealed by tracer-tracer scatter plots, J. Geophys. Res., 102, 13 119-13 134, (96JD03 715), 1997.

Williamson, D. L.: Semi-Lagrangian moisture transport in the NMC spectral model, Tellus, 42A, 413-428, 1989.

Woodman, R. and Rastogi, P.: Evaluation of effective eddy diffusive coefficients using radar observations of turbulence in the stratosphere, Geophys. Res. Lett., 11, 243-246, 1984. 